\title{
Numerical Modeling of Shock-Wave Interaction with a Laminar Boundary Layer during Hyper- sonic Flow around Models with a Kink
}

\author{
Sergey T. Surzhikov \\ Ishlinskyi Institute for Problems in Mechanics Russian Academy of Sciences (IPMech RAS), \\ Moscow, 119526, Russia \\ N.L. Dukhov All-Russian Scientific Research Institute of Automatics \\ surg@ipmnet.ru

\begin{abstract}
A series of experimental data obtained at the Calspan-UB Research Center (CUBRC), and related numerical calculations performed by participants of the working group of testing computer codes, were used to validate the author's computer code PERAT-2D.

The problems of flowing by hypersonic flows of molecular nitrogen of a sharp and blunt cone with a kink are considered, as well as the problem of flowing around a hollow cylinder with a sharp edge and an expanding skirt.
\end{abstract}

Keywords: hypersonic flow, the interaction of a shock wave with a boundary layer, validation of an author's computer code
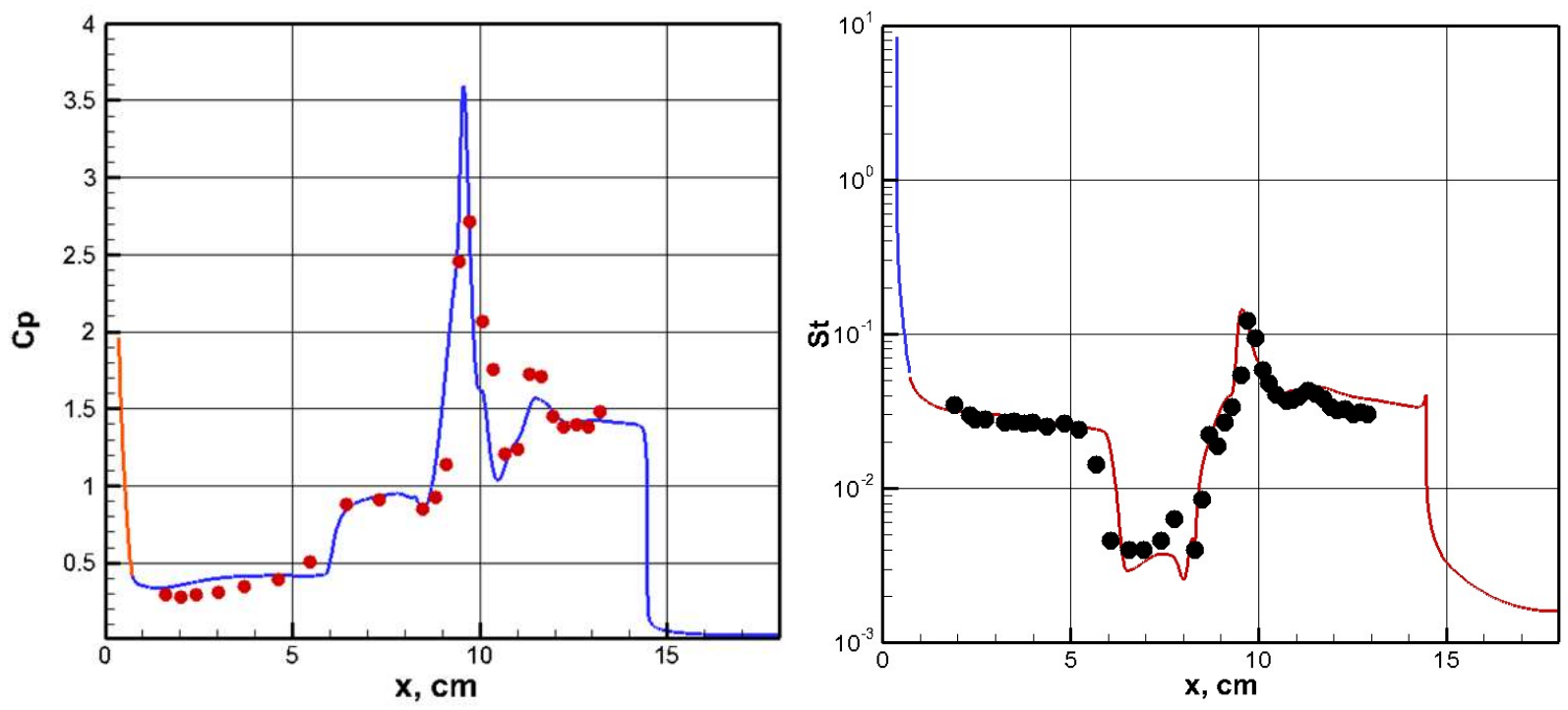

Distributions of the pressure coefficient $C_{p}$ and the heat transfer coefficient St along the surface of a hollow cylinder with a skirt for test task Run \# 31 [Holden M.S. et al., RTO-TR-AVT-007-03]. Comparison of experimental and computed data 


\title{
Численное моделирование ударно-волнового взаимодействия с ламинарным пограничным слоем при гиперзвуковом обтекании моделей с изломом образующей
}

\author{
С.Т. Суржиков \\ Институт проблем механики им. А.Ю. Иилинского Российской академии наук \\ Россия, Москва, 119526, проспект Вернадского, д. 101-1 \\ Всероссийский научно-исследовательский институт автоматики им. Н.Л. Духова \\ surg@ipmnet.ru
}

\begin{abstract}
Аннотация
Серия экспериментальных данных, полученных в Calspan-UB Research Center (CUBRC), и сопутствующих численных расчетов, выполненных участниками рабочей группы тестирования компьютерных кодов, использованы для валидации авторского компьютерного кода PERAT-2D.

Рассмотрены задачи обтекания острого и затупленного конуса с изломом образующей, а также задача обтекания полого цилиндра с острой кромкой и расширяющейся юбкой гиперзвуковыми потоками молекулярного азота.

Ключевые слова: гиперзвуковое обтекание, взаимодействие ударной волны с пограничным слоем, валидация авторского компьютерного кода
\end{abstract}

\section{1. Введение}

База экспериментальных данных, полученная в Calspan-UB Research Center (CUBRC) при изучении особенностей гиперзвукового обтекания осесимметричных моделей с изломом образующей, была положена в основу серии валидирующих расчетов в рамках специально организованной рабочей группы по сравнению расчетных и экспериментальных данных [1-20]. Аналогичные исследования параллельно проводились и другими научными группами [22-28]. Результаты детальных экспериментальных и расчетных исследований, выполненных в ЦАГИ под руководством В.Я. Борового, приводятся в монографии [25].

Часть полной матрицы экспериментальных данных, использованных для валидации компьютерных кодов представлена в табл. 1. Для некоторых экспериментальных точек имеется несколько вариантов исходных данных. Согласно [2], часть этих данных была уточнена в экспериментах, а часть данных соответствует расчетному предсказанию параметров в набегающем потоке, в том числе с учетом неравновесных физико-химических процессов.

Подробный анализ указанных экспериментальных данных и результатов выполненного расчетного исследования показали ряд принципиальных особенностей ударно-волнового взаимодействия с ламинарным пограничным слоем, а также развития отрывной зоны навстречу потоку в окрестности излома образующей обтекаемой модели.

Одним из важных результатов, касающихся численного моделирования указанных задач, оказался вывод о чрезвычайной чувствительности расчетных данных к качеству расчетного метода и степени подробности используемых расчетных сеток. Так, например, в работе [3] для решения этих задач применялись коды WIND (NPARC Alliance) и DPLR (NASA Ames 
Research Center). Экспериментальные данные для двух аэродинамических моделей были выбраны для тестирования: полый цилиндр с расширяющейся под углом $30^{0}$ юбкой и двойной конус с углами раствора $25^{0}$ и $55^{\circ}$. Один из конусов был острым, а второй имел радиус скругления 0.635 см. Расчеты проводились на одних и тех же структурированных расчетных сетках разной подробности (например, для двойного конуса: $128 \times 48,256 \times 96,512 \times 192,1024 \times 384$, $2048 \times 768)$.

Таблица 1

Исходные данные тестов по гиперзвуковому обтеканию

\begin{tabular}{|c|c|c|c|c|c|c|}
\hline Run \# & $V_{\infty}, \mathrm{cm} / \mathrm{c}$ & $\rho_{\infty}, \Gamma / \mathrm{cm}^{3}$ & $p_{\infty}$, эрг $/ \mathrm{cm}^{3}$ & $T_{\infty}, \mathrm{K}$ & $\mathrm{M}$ & Библиография \\
\hline $11 \mathrm{a}$ & $2.609 \times 10^{5}$ & $0.5066 \times 10^{-06}$ & 194 & 128.9 & 11.1 & \\
\hline $11 \mathrm{~b}$ & $2.485 \times 10^{5}$ & $0.5866 \times 10^{-06}$ & 171.9 & 98.7 & 12.3 & $2002-581$ \\
\hline $14 \mathrm{a}$ & $2.432 \times 10^{5}$ & $0.7937 \times 10^{-06}$ & 367.9 & 156.1 & 9.5 & \\
\hline $14 \mathrm{~b}$ & $2.327 \times 10^{5}$ & $0.7506 \times 10^{-06}$ & 254.3 & 114.19 & 10.7 & \\
\hline $14 \mathrm{c}$ & $2.304 \times 10^{5}$ & $0.8810 \times 10^{-06}$ & 318.8 & 120.4 & 10.3 & \\
\hline 24 & $2.614 \times 10^{5}$ & $1.3700 \times 10^{-06}$ & 619 & 152. & 10.4 & \\
\hline $28 \mathrm{a}$ & $2.522 \times 10^{5}$ & $0.7267 \times 10^{-06}$ & 299.9 & 139. & 10.5 & \\
\hline $28 \mathrm{~b}$ & $2.664 \times 10^{5}$ & $0.6345 \times 10^{-06}$ & 360.6 & 185.6 & 9.59 & \\
\hline $28 \mathrm{c}$ & $2.538 \times 10^{5}$ & $0.7372 \times 10^{-06}$ & 306.4 & 140. & 10.5 & \\
\hline $35 \mathrm{a}$ & $2.577 \times 10^{5}$ & $0.6082 \times 10^{-06}$ & 185.5 & 102.7 & 12.5 & \\
\hline $35 \mathrm{~b}$ & $2.713 \times 10^{5}$ & $0.5515 \times 10^{-06}$ & 227.4 & 138.9 & 11.3 & \\
\hline 31 & $2.623 \times 10^{5}$ & $0.5670 \times 10^{-06}$ & 180.6 & 107.3 & 12.4 & \\
\hline 32 & $2.574 \times 10^{5}$ & $0.7576 \times 10^{-06}$ & 320.6 & 142.5 & 10.6 & \\
\hline 36 & $2.586 \times 10^{5}$ & $1.1080 \times 10^{-06}$ & 495.8 & 150.7 & 10.3 & \\
\hline
\end{tabular}

Помимо математической оценки точности получаемых численных результатов с использованием расчетной технологии последовательности расчетных сеток, в работе [3] проводилось сравнение получаемых данных по коэффициентам давления

$$
C_{p}=\frac{p-p_{\infty}}{0.5 \rho_{\infty} V_{\infty}^{2}}
$$

и теплообмена (коэффициент Стантона)

$$
C_{h}=\mathrm{St}=\frac{q_{w}}{0.5 \rho_{\infty} V_{\infty}^{3}}
$$

где $p, q_{w}$ - давление и плотность конвективного теплового потока на поверхности тела; $p_{\infty}, \rho_{\infty}, V_{\infty}$ - давление, плотность и скорость набегающего потока.

Указанные выше расчетные коды показали достаточно близкие результаты. Однако, использование разных расчетных сеток привело к качественно различающимся данным.

Исследованные аэродинамические модели оказались очень удобными для получения наглядных качественных и количественных экспериментальных данных по ударно-волновому взаимодействию с пограничным слоем. Расчетные области и сильно разреженные сетки, использованные в данной работе показаны на рис. 1.

Как при обтекании полого цилиндра с заостренными кромками и юбкой, так и при обтекании двойного конуса в экспериментах у поверхности фиксировались 4 характерные области течения. Для примера, на рис. 2 показаны распределения коэффициентов $C_{p}(a)$ и $C_{h}=\mathrm{St}$ вдоль поверхности двойного затупленного конуса (см. рис. 1, в). Первая область отвечает обтеканию сферического затупления, переходящего в конус с углом раствора $25^{0}$. 
Во второй зоне наблюдается рост давления и падения плотности конвективного теплового потока в зоне отрывного течения перед и над изломом образующей. Третья зона, максимального роста $C_{p}$ и $C_{h}$, соответствует местоположению падения головной отошедшей ударной волны на пограничный слой. И четвертая зона отвечает давлению и теплообмену за местом взаимодействия ударной волны с пограничным слоем.

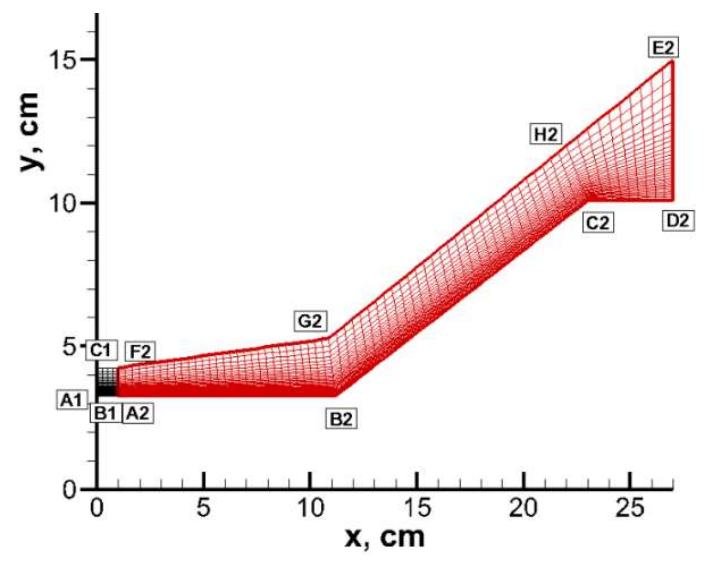

(a)
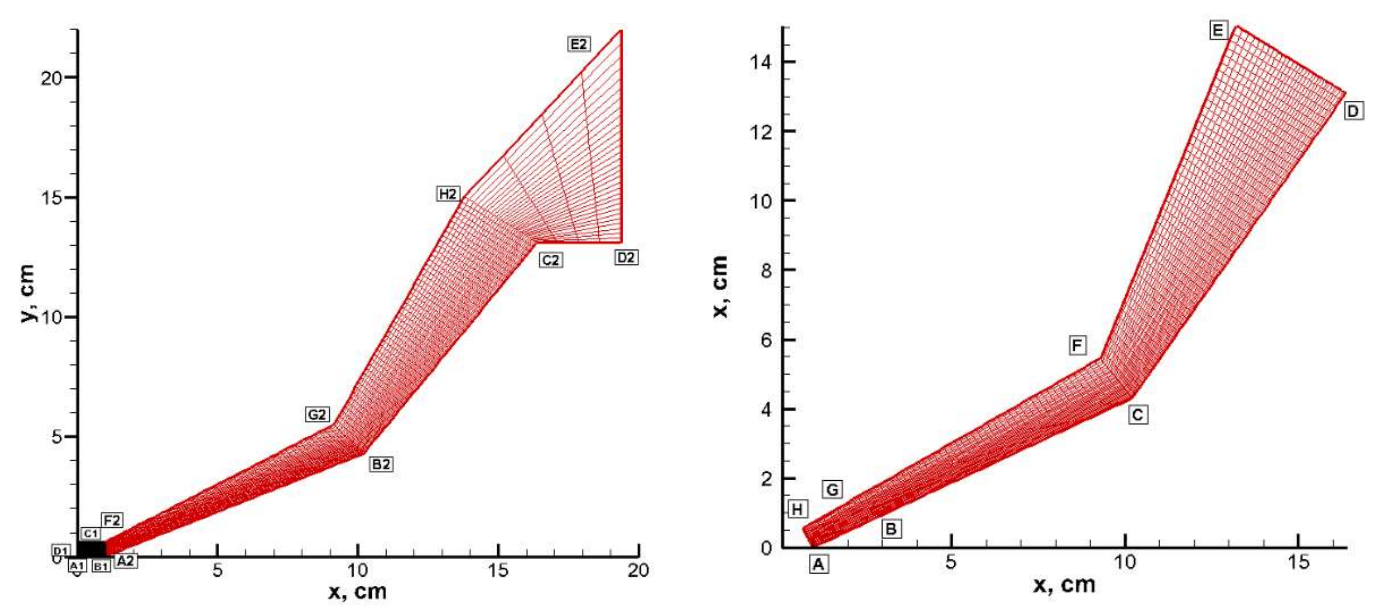

(б)

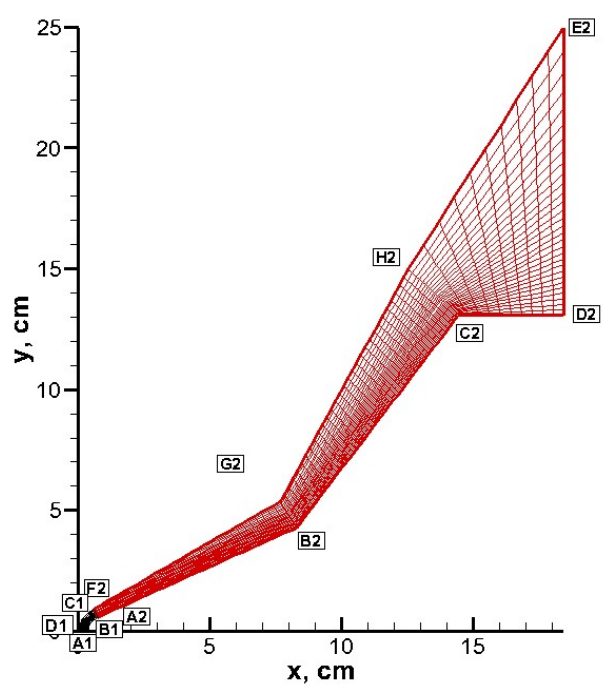

(b)

Рис. 1. Геометрия и расчетная область полого цилиндра с юбкой $(a)$, двойного острого конуса (б) и двойного затупленного конуса (в) 
Было установлено, что важнейшим показателем качества получаемого решения является точность численного предсказания протяженности отрывной зоны, местоположения области взаимодействия ударной волны с пограничным слоем и абсолютные значения коэффициентов $C_{p}$ и $C_{h}$ в указанных зонах. Наиболее чувствительной к расчетным сеткам оказалась расчетная длина отрывной зоны и значения коэффициентов $C_{p}$ и $C_{h}$ в ней.
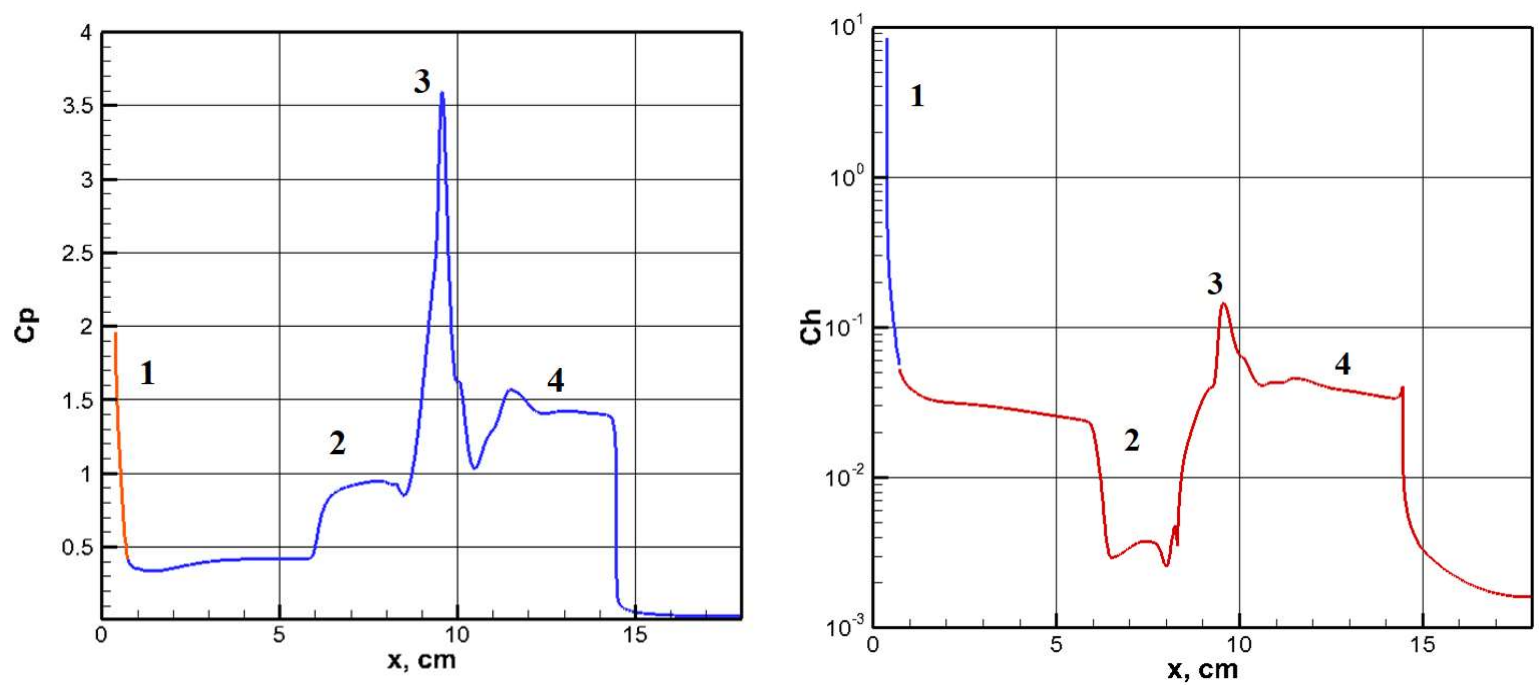

Рис. 2. Характерные элементы поверхностных распределений коэффициентов давления $C_{p}$ и теплообмена $C_{h}$ при гиперзвуковом обтекании моделей

В работе [3] были выполнены расчеты по коду DPLR не только с использованием модели совершенного газа, но и с учетом неравновесных физико-химических процессов. Было показано некоторое, но не принципиально важное влияние на результаты расчетов. Применение DSMC расчетных кодов дало более заметное расхождение с экспериментальными данными, что явилось предметом подробного анализа в $[1,2]$.

Итоги работы по экспериментальному исследованию гиперзвуковых течений и по валидации компьютерных кодов, выполненных при поддержке Calspan-UB Research Center (CUBRC) и Air Force Office of Scientific Research (AFOSR), подведены в работе [1].

Значительные усилия были предприняты по достоверному определению параметров потока на испытуемых моделях. В указанной работе дан подробный анализ каждого экспериментального теста и соответствующих валидирующих расчетов. Также дан анализ расчетных данных, представленных в работах [5-12].

Как уже отмечалось, было продемонстрировано в целом хорошее согласие расчетных и экспериментальных данных. Вместе с тем, анализ результатов расчетов разных авторов показывает, что не всегда одни и те же коды дают полное согласие с экспериментом. Здесь особо заметим, что достоверность экспериментальных данных в данном случае не подвергается сомнению. Во-первых, следует отметить высокую тщательность исполнения этих экспериментов [1]. Во-вторых, серия валидационных расчетов разных авторов, выполненных также с высокой тщательностью, подтверждают de-facto соответствие полученных экспериментальных данных используемым исходным данным в набегающем потоке и геометрическим параметрам моделей [13-15]. Тем не менее, предпринятые усилия по валидации компьютерных кодов на примерах экспериментов CUBRC привели к некоторому уточнению экспериментальных данных [2]. Например, при анализе эксперимента Run\#11 очень хорошее согласие с экспериментом в области отрывного течения демонстрируют расчетные данные Като и Таннехилла [8]. При этом расчеты Кандлера [9] и Ноффо [10] дают более протяженную отрывную зону. В то же время, расчеты Кандлера и Ноффо прекрасно совпадают с экспериментальными данными Run\#28. 
Ниже, на примере анализа расчетных результатов данной работы, будет показано, что указанные расхождения вполне могут быть обусловлены различиями в топологии используемых сеток, что еще раз подтверждает высокую чувствительность рассчитанных данных к используемому методу и конечно-разностным сеткам.

\section{2. Двухмерная численная модель Навье-Стокса ламинарного обтекания аэродинамических моделей гиперзвуковым потоком совершенного газа. Компьютерный код PERAT-2D}

Расчетная модель вязкого и теплопроводного совершенного газа основана на уравнении неразрывности, системе уравнений Навье-Стокса, уравнении сохранения энергии, термического уравнения состояния идеального газа Клайперона-Менделеева:

$$
\begin{gathered}
\frac{\partial \rho}{\partial t}+\operatorname{div}(\rho \mathbf{V})=0 \\
\frac{\partial \rho u}{\partial t}+\operatorname{div}(\rho u \mathbf{V})=-\frac{\partial p}{\partial x}-\frac{2}{3} \frac{\partial}{\partial x}(\mu \operatorname{div} \mathbf{V})+\frac{\partial}{\partial y}\left[\mu\left(\frac{\partial u}{\partial y}+\frac{\partial v}{\partial x}\right)\right]+2 \frac{\partial}{\partial x}\left(\mu \frac{\partial u}{\partial x}\right) \\
\frac{\partial \rho v}{\partial t}+\operatorname{div}(\rho v \mathbf{V})=-\frac{\partial p}{\partial y}-\frac{2}{3} \frac{\partial}{\partial y}(\mu \operatorname{div} \mathbf{V})+\frac{\partial}{\partial x}\left[\mu\left(\frac{\partial u}{\partial y}+\frac{\partial v}{\partial x}\right)\right]+2 \frac{\partial}{\partial y}\left(\mu \frac{\partial v}{\partial y}\right) \\
\rho c_{p} \frac{\partial T}{\partial t}+\rho c_{p} \mathbf{V g r a d} T=\frac{\partial p}{\partial t}+\mathbf{V} \operatorname{grad} p+\operatorname{div}(\lambda \operatorname{grad} T)
\end{gathered}
$$

где $x, y$ - декартовы координаты; $\mathbf{V}=(u, v)$ - скорость потока и ее проекции на оси $x$ и $y ; \rho, p$ - плотность и давление; $\mu$ - динамический коэффициент вязкости; $c_{p}$ - удельная теплоемкость при постоянном давлении; $T$ - температура; $\lambda$ - коэффициент теплопроводности. Уравнение сохранения энергии здесь записано в неконсервативной форме относительно температуры (в форме уравнения Фурье-Кирхгофа).

Система уравнений (1)-(4) используется совместно с уравнением состояния идеального газа

$$
p=\rho \frac{R_{0}}{M_{\Sigma}} T
$$

где $R_{0}=8.314 \times 10^{7}$ эрг/(моль $\left.\cdot \mathrm{K}\right)-$ универсальная газовая постоянная; $M_{\Sigma}=29$ г/моль - молекулярный вес газа.

Граничные условия задают невозмущенный набегающий поток (см. рис. 2)

$$
s(x, y): u=u_{\infty}, \quad v=0, T=T_{\infty}, \quad p=p_{\infty}, \rho=\rho_{\infty}
$$

и условия второго рода для изменения функций вдоль потоковой координаты $\xi$ на выходе из расчетной области

$$
x=L: \quad \frac{\partial u}{\partial \xi}=\frac{\partial v}{\partial \xi}=\frac{\partial T}{\partial \xi}=\frac{\partial \rho}{\partial \xi}=0
$$

На поверхности обтекаемого тела задаются условия прилипания

$$
\eta=0: \quad u=v=0, \quad T=T_{w}, \quad \frac{\partial p}{\partial \eta}=0,
$$

где $\eta$ - нормальная к поверхности координатная линия. 
Данная расчетная модель, реализованная в компьютерном коде PERAT-2D (Perfect Aero Thermodynamics - Two-Dimensional), является упрощенной версией модели, на основе которой был создан компьютерный код NERAT-2D (Non-Equilibrium Radiative AeriThermodynamics Two-Dimensional), прошедший большую серию тестирования на примерах решения задач неравновесной аэрофизики [29].

Алгоритмически реализованы три версии кода PERAT-2D:

1) Двухблочный расчетный код PERAT-2D-2BL без возможности автоматического удвоения сеток. Максимальный размер расчетных сеток достиг величины 703 × 33 (1-й блок) $+703 \times 1505$ (2-й блок).

2) Одноблочный расчетный код PERAT-2D-1BL с автоматическим удвоением расчетной сетки. Максимальный достигнутый размер расчетной сетки: $4001 \times 385$.

3) Одноблочный расчетный код PERAT-D2D-1BL с удвоенной точностью и с автоматическим удвоением сетки.

Во всех компьютерных кодах реализованы встроенные программы построения расчетных сеток с произвольной степенью сжатия к поверхности. Дополнительное сжатие в области ударной волны не применялось.

Так же, как и в [3], расчеты каждого варианта производились на последовательности расчетных сеток. При использовании кода PERAT-2D-2BL расчеты производились без использования многосеточной технологии.

После получения решения на заданной сетке производилась вариация степени сгущения вблизи поверхности. Дополнительное численное исследование проводилось при изменении координат угловых точек В2, G2 (рис. 1, a), В2, G2, С2, Н2 (рис. 1, б (слева), 1, в), С, F (рис. 1, б (справа)). Расчеты обтекания острого двойного конуса выполнялись по двум расчетным схемам (рис. 1, б): с участком невозмущенного набегающего потока и без такого участка.

\section{3. Метод численного интегрирования}

Как уже отмечалось, компьютерный код PERAT-2D является упрощенной версией компьютерного кода NERAT-2D. Главные изменения коснулись перехода к модели совершенного газа и, тем самым, значительной экономии используемой памяти (без использования многоблочной технологии параллельных вычислений). Численный алгоритм расчетов остался прежним.

Система уравнений (1)-(4) интегрировалась до сходимости искомых сеточных с точностью $10^{-4}$. Определялась относительная ошибка расчета каждой функции по всему полю течения. Уравнения газовой динамики (уравнение неразрывности и два уравнения НавьеСтокса) интегрировались с применением AUSM конечно-разностной схемы второго порядка точности [36]. Уравнение теплопроводности интегрировались с использованием неявной конечно-разностной схемы второго порядка точности по пространству и времени. Применение неявных конечно-разностных схем к решению уравнения теплопроводности значительно повышало эффективность вычислительной процедуры. Подчеркнем, что дополнительные численные ограничители и какие-либо виды искусственной вязкости не использовались.

\section{4. Результаты численного моделирования}

Исходные данные для выполненных расчетов приведены в табл. 1. В данной работе представлены результаты расчетов полого цилиндра с юбкой (варианты Run\#11 и Run\#14), заостренного двойного конуса (Run\#35a) и затупленного двойного конуса (Run\#31 и Run\#32).

Прежде, чем обсудить результаты валидирующих расчетов, подчеркнем сильное влияние используемых конечно-разностных схем для всех без исключения рассчитанных вариантов. Достаточно важным оказался выбор и формы внешней границы расчетной области (рис. 1).

На рис. 3 показаны распределения коэффициентов давления и теплообмена для эксперимента Run\#35, полученные с использованием конечно-разностных сеток разной подробности. 
Это типичные результаты для всех рассчитанных вариантов по влиянию подробности сеток на степень совпадения расчетных данных с экспериментальными.
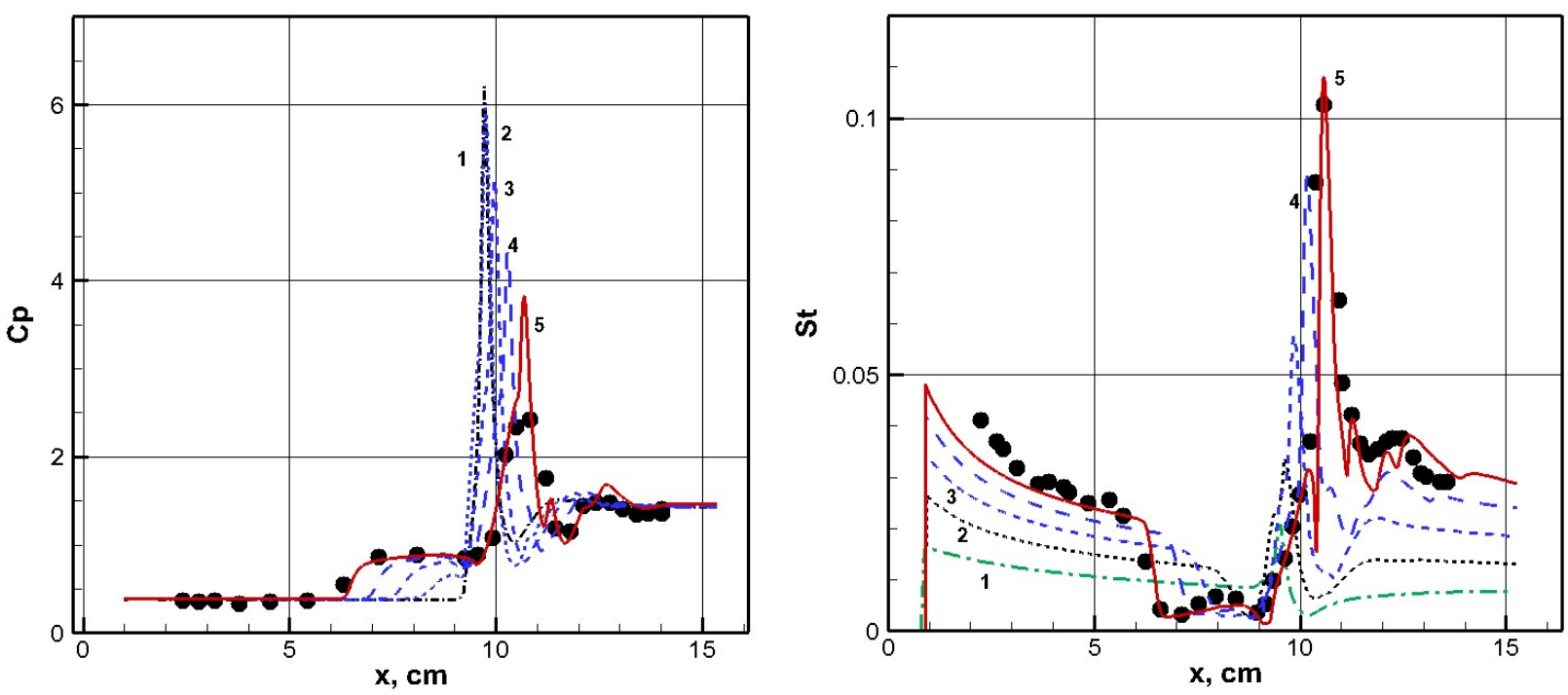

Рис. 3. Распределение коэффициента давления (a) и коэффициента теплообмена (b) при обтекании затупленного двойного конуса в эксперименте Run\#35. Цифры у кривых: 1 - расчетная сетка $251 \times 25$ (вдоль и поперек поверхности); 2 - $501 \times 49 ; 3-1001 \times 97 ; 4-2002 \times 193 ; 5-4001 \times 385$. Дискретные точки - экспериментальные данные [1]

Распределения газодинамических функций в эксперименте с обтеканием полого цилиндра с юбкой (Run\#11) показаны на рис. 4 и 5. В расчетах учитывалась область невозмущенного течения до передней кромки полого цилиндра.

Отметим основные особенности поля течения. Ударная волна, генерируемая передней кромкой цилиндра, взаимодействует с пограничным слоем на расстоянии $x \sim 15.5$ см. У внешней поверхности полого цилиндра формируется пограничный слой, который отделен от головной ударной волны областью невязкого течения. Пограничный слой развивается от кромки вдоль поверхности цилиндра вплоть до достижения области возвратно-вихревого движения газа в зоне отрыва потока вблизи излома образующей.

Существование области отрывного течения над изломом образующей является важной особенностью взаимодействия ударной волны с пограничным слоем над конической юбкой. Здесь возникаем область повышенного давления, которая и является причиной разворота потока в пограничном слое навстречу основному потоку. Из рис. 4, 6 видно, что область повышенного давления распространяется от места взаимодействия ударной волны с пограничным слоем $(x \sim 15.5$ см) навстречу потоку до расстояния $x \sim 9$ см, где заканчивается область возвратного движения. Зона возвратного движения хорошо видна на рис. 4, 2.

Много информации по структуре течения можно получить из анализа поля модуля градиента плотности на рис. 5. Здесь хорошо видны внешняя и пристеночная области пограничного слоя на внешней поверхности цилиндра и фронт головной ударной волны. Над конической юбкой наблюдаются скачек отраженной волны, скачек отрывной зоны и скачек присоединения, а также пограничный слой.

Примечательна зона повышенной температуры ( $12 \leq x \leq 15$ см), формируемая над отрывным течением и область с повышенной температурой за отраженной ударной волной.

На рис. 6 даны распределения давления и плотности конвективного теплового потока вдоль поверхности для номинальной скорости набегающего потока $V_{\infty}=2.6 \mathrm{\kappa M} / \mathrm{c}(a)$, а также для уменьшенной $\left(V_{\infty}=2.2 \mathrm{\kappa м} / \mathrm{c}\right)$ и увеличенной скорости $\left(V_{\infty}=2.8 \mathrm{\kappa м} / \mathrm{c}\right)$. Эти расчеты были выполнены с целью оценить влияние возможной неоднородности в набегающем потоке. 


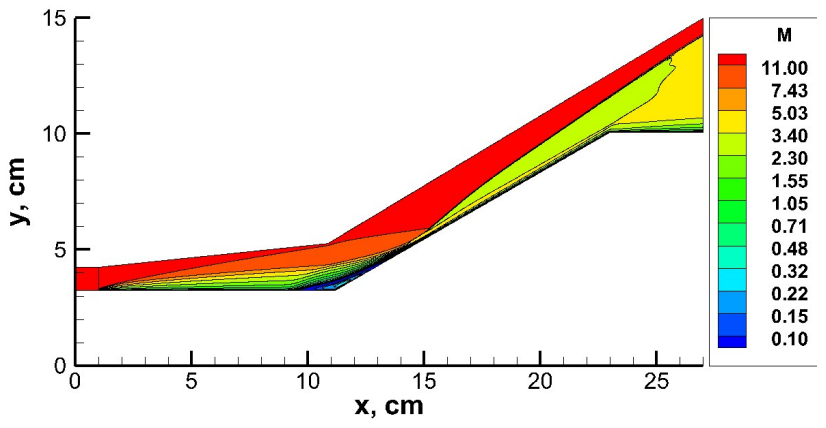

(a)

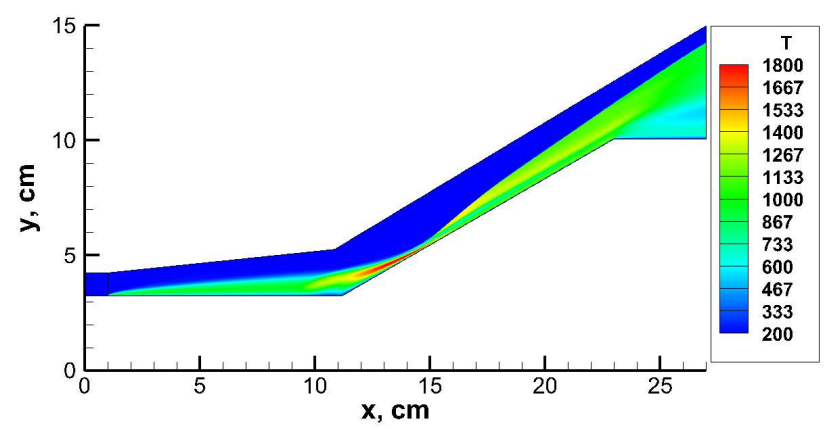

(B)

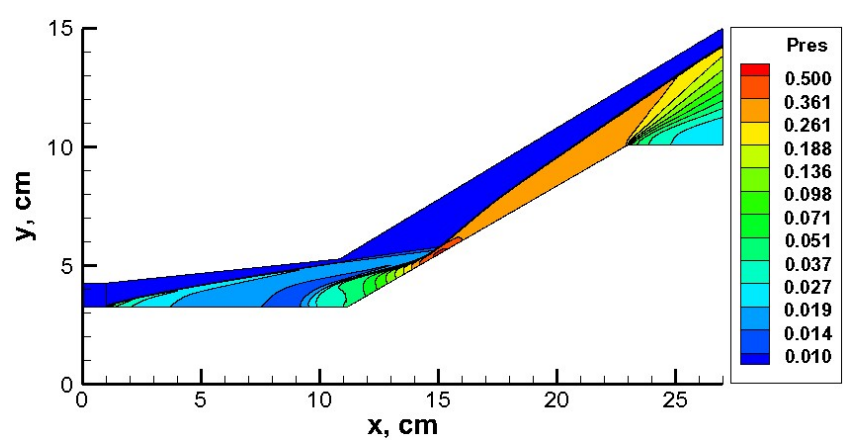

(б)

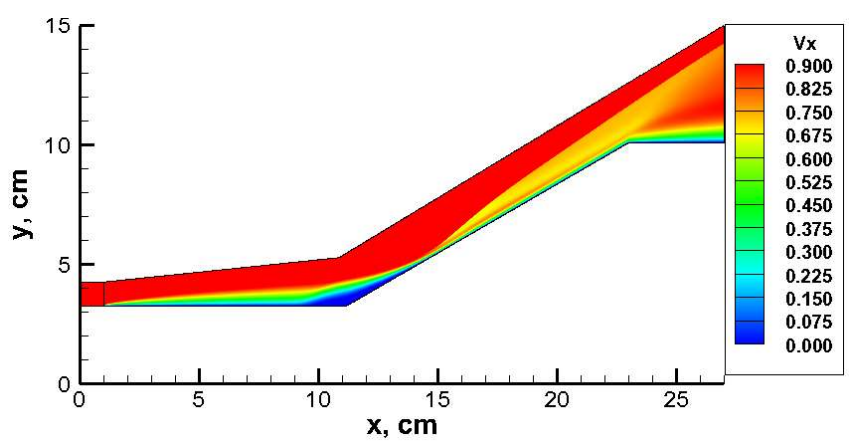

(2)

Рис. 4. Поле чисел Маха $(a)$; давления ( Pres $\left.=p / \rho_{\infty} V_{\infty}^{2}\right)(б)$; температуры (в К) (в) и продольной скорости $V_{x}=u / V_{\infty}$ (2) для тестовой задачи Run\#11 (расчетная сетка $703 \times 1505$, коэффициент сгущения расчетной сетки к поверхности $\beta=0.8$ )

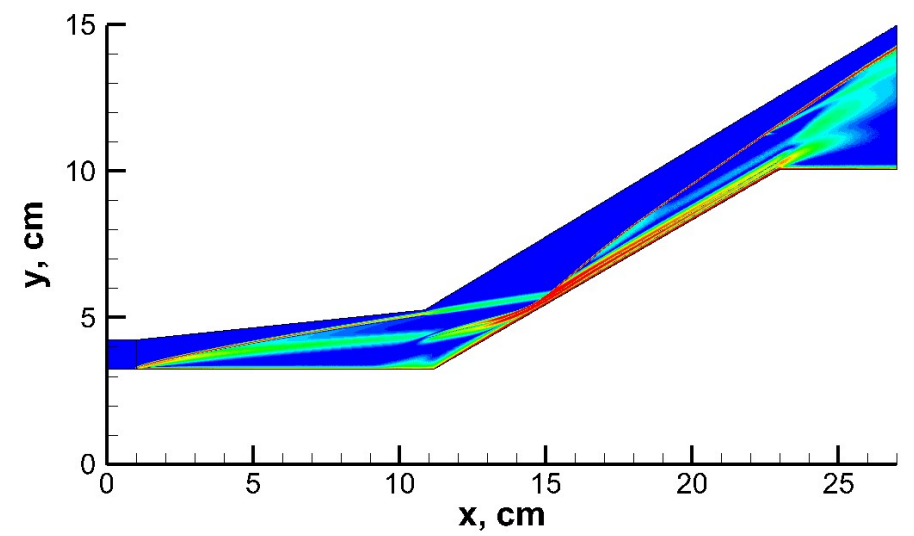

Рис. 5. Поле модуля градиента плотности для тестовой задачи Run\#11 (расчетная сетка $703 \times 1505$, коэффициент сгущения расчетной сетки к поверхности $\beta=0.8$ )

Изменение скорости набегающего потока приводит к закономерному уменьшению (с уменьшением $V_{\infty}$ ) или увеличению давления и теплового потока на поверхности юбки. Для всех рассчитанных вариантов имеется заметное расхождение давления в месте взаимодействия падающей ударной волны с пограничным слоем (при $x \sim 15$ см). В работах других авторов такое расхождение также наблюдается.

В целом отметим, что локальное повышение давления в зоне отрывного течения и над поверхностью юбки хорошо совпадают с экспериментальными значениями при номинальной скорости набегающего потока.

Распределение плотности теплового потока также хорошо коррелирует с экспериментальными данными, включая зону энтропийного течения $(x \sim 11 \mathrm{~cm})$, где заметно локальное снижение плотности теплового потока. 

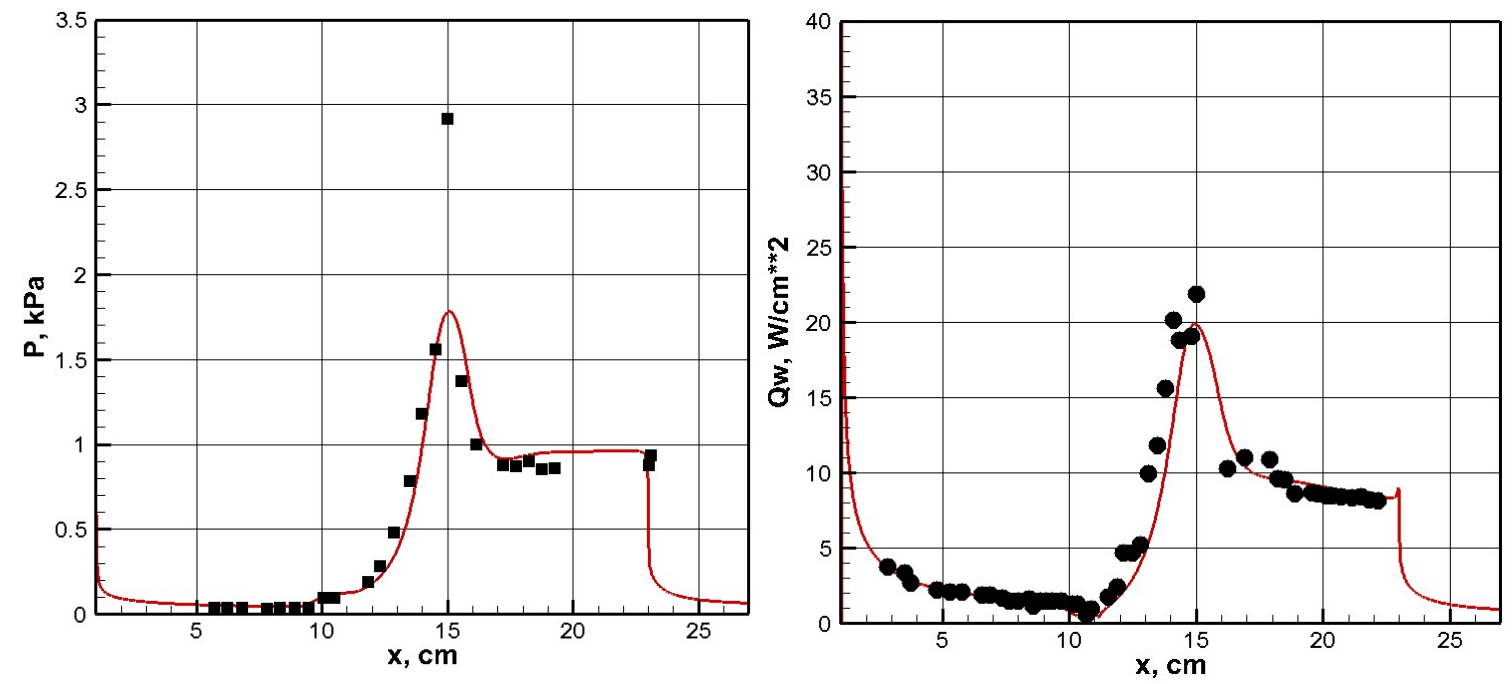

(a)
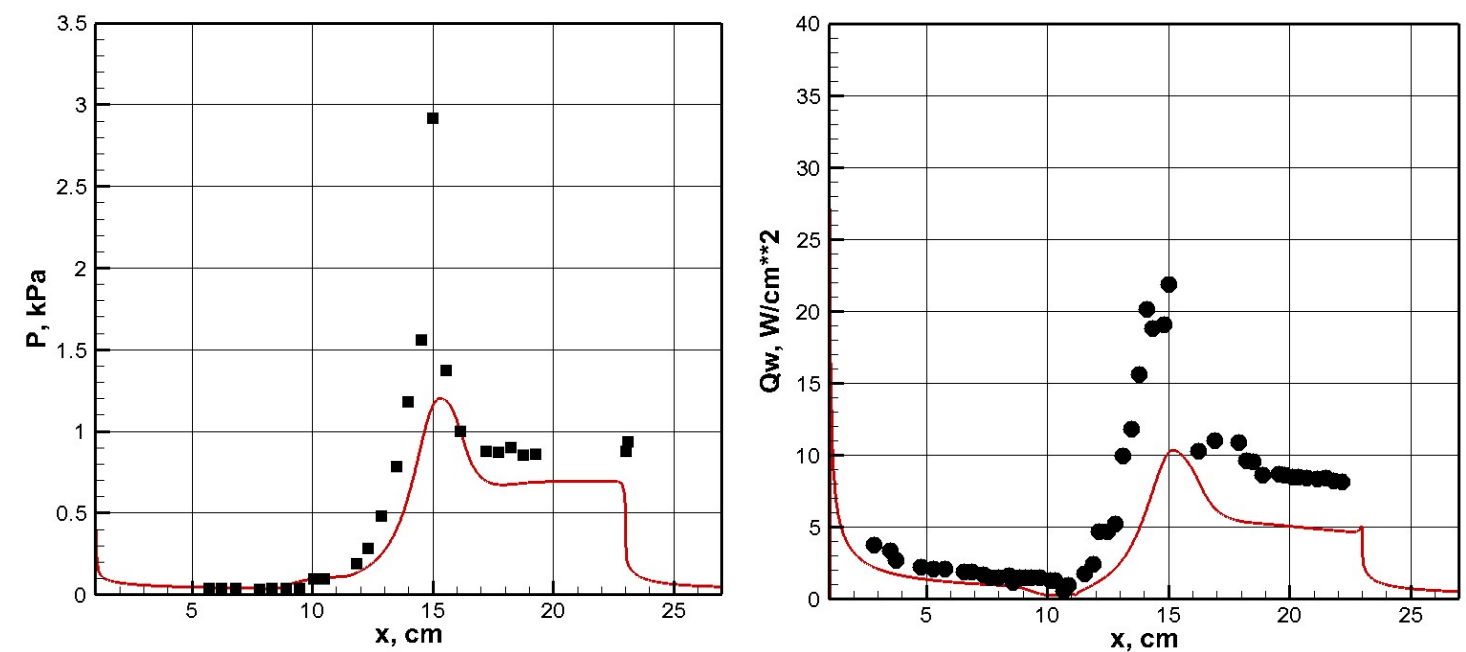

(б)
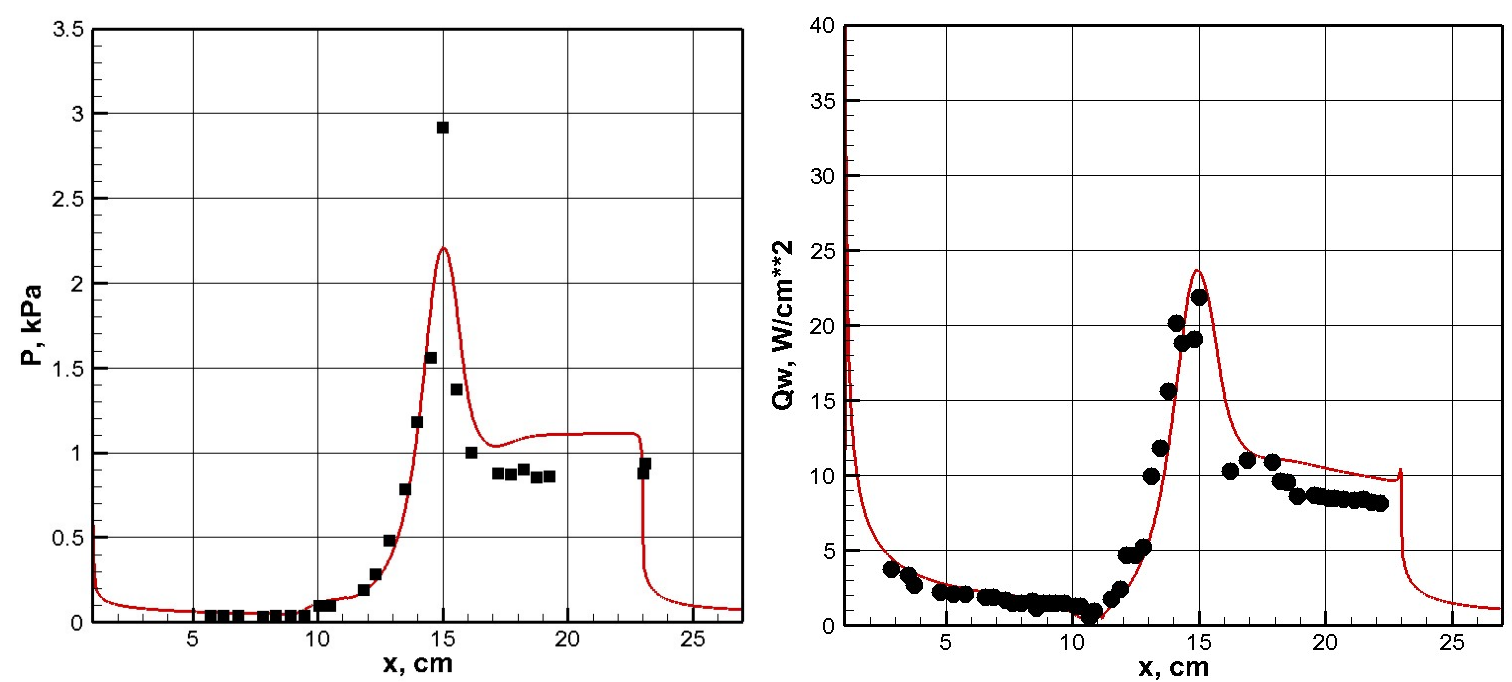

(B)

Рис. 6. Распределение давления (слева) и плотности конвективного теплового потока (справа) вдоль поверхности полого цилиндра с юбкой для тестовой задачи Run\#11 (расчетная сетка $703 \times 1505$, коэффициент сгущения расчетной сетки к поверхности $\beta=0.8 ;(a)-V_{\infty}=2.6 \mathrm{\kappa м} / \mathrm{c}$, $\left.(б)-V_{\infty}=2.2 \mathrm{\kappa m} / \mathrm{c},(8)-V_{\infty}=2.8 \mathrm{\kappa m} / \mathrm{c}\right)$ 
На рис. 7-9 представлены результаты расчетов обтекания полого цилиндра с конической юбкой для теста Run\#14. Отличием от ранее рассмотренного варианта является увеличение плотности и давления в набегающем потоке при некотором снижении скорости, что приводит к уменьшению числа Маха до М 10 .

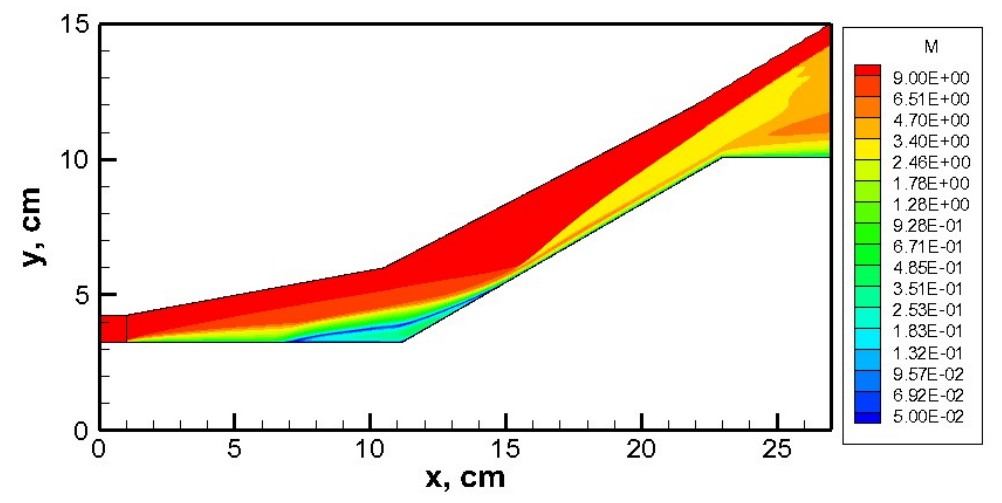

(a)

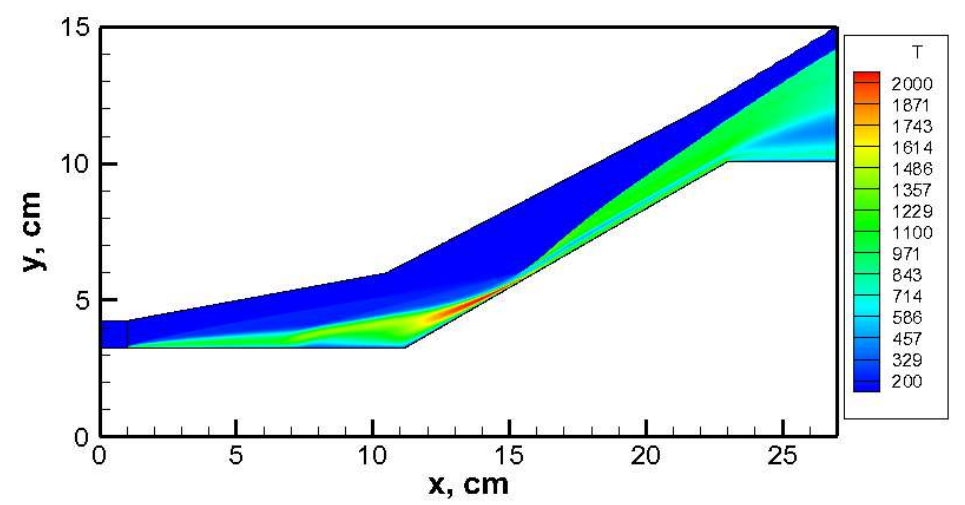

(б)

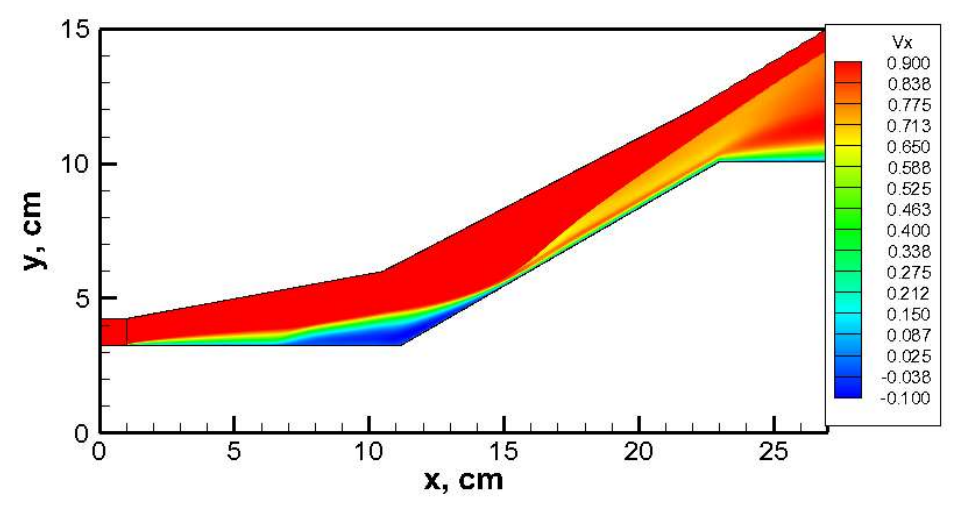

(в)

Рис. 7. Поле чисел Маха $(a)$, температуры (в К) (б) и продольной скорости $V_{x}=u / V_{\infty}$ для тестовой задачи Run\#14 (расчетная сетка $703 \times 1505$, коэффициент сгущения расчетной сетки к поверхности $\beta=0.8$ )

В целом, что структура поля течения оказывается подобной выше, однако, обратим внимание на существенно более протяженную область отрывного течения над изломом образующей. На рис. 9 видно, что протяженность области пониженного давления и повышенной плотности конвективного теплового потока, ассоциируется с зоной отрывного течения составляет порядка 5 см. Совпадение с экспериментальными данными вполне хорошее, за исключением максимального значения плотности теплового потока. Заметим, что в предыдущем варианте наблюдалось аналогичное расхождение для максимального давления. 

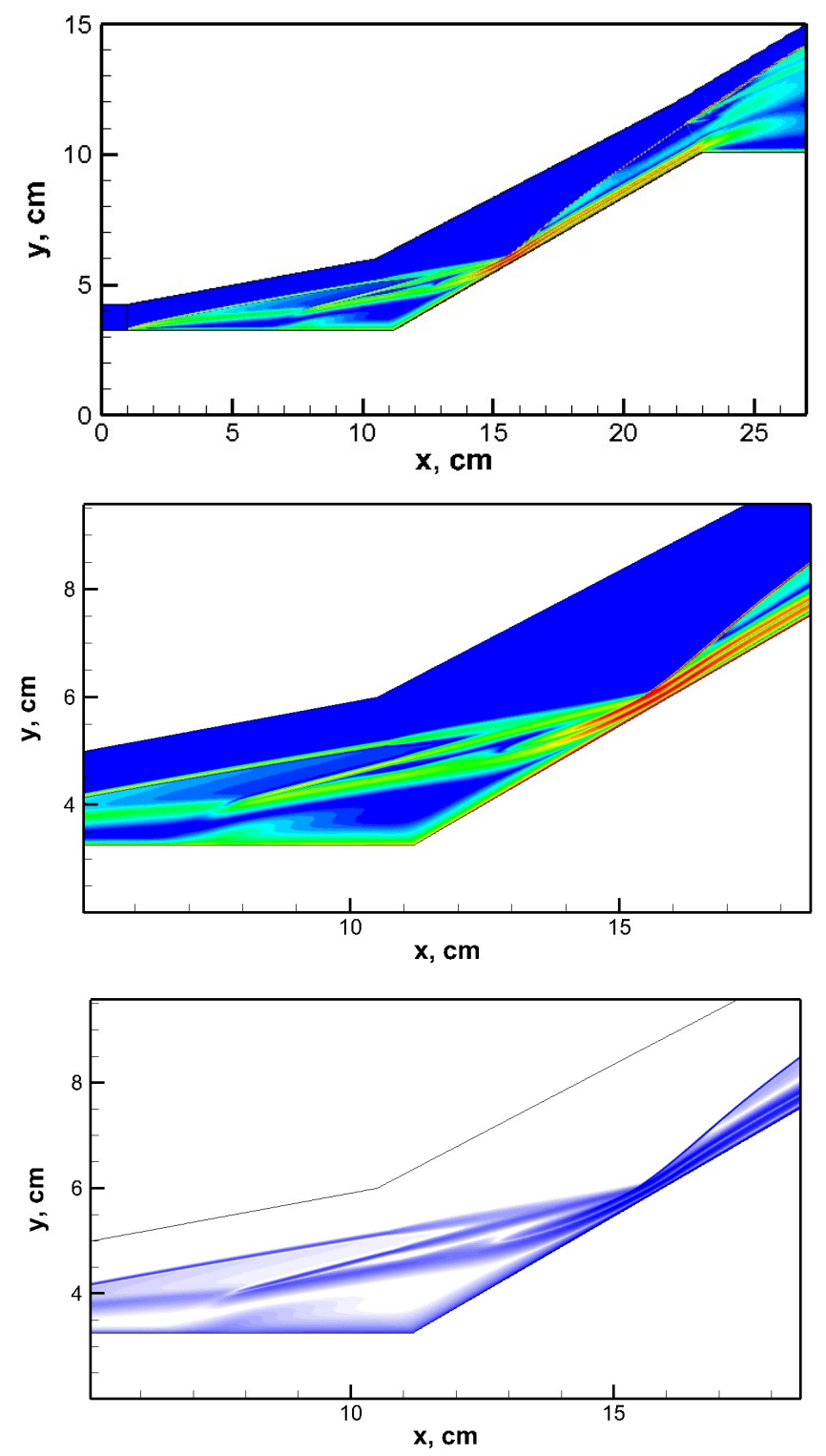

Рис. 8. Поле модуля градиента плотности для тестовой задачи Run\#14 (расчетная сетка $703 \times 1505$, коэффициент сгущения расчетной сетки к поверхности $\beta=0.8$ )
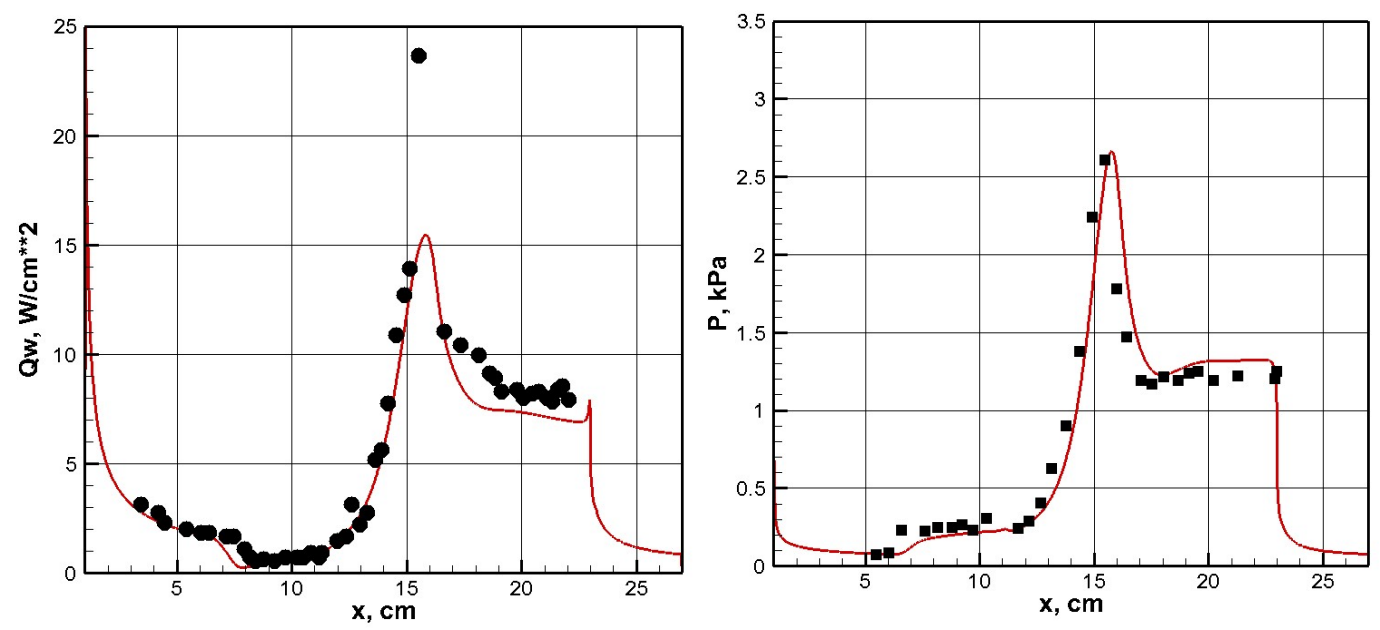

Рис.9. Распределение плотности конвективного теплового потока и давления вдоль поверхности полого цилиндра с юбкой для тестовой задачи Run\#14 
Отметим еще одну особенность структуры поля течения в тестовой задаче Run\#14. Поскольку размеры отрывной зоны стали больше, наблюдается вязкое взаимодействие скачка отрыва с отрывной зоной, такое, что указанный скачек отрыва достигает головной ударной волны ранее, чем происходит взаимодействие головного скачка с пограничным слоем на конической юбке.

B тестовом варианте Run\#31 изучается взаимодействие ударной волны с пограничным слоем у поверхности двойного затупленного конуса, так что проявляется еще влияние энтропийного слоя, образованного при обтекании головного затупления.

На рис. 10-13 показаны профили газодинамических функций, полученные при разных степенях сгущения расчетной сетки к поверхности конуса и конфигурации внешней границы расчетной области. Показанные профили дают представление о степени такого влияния. Визуально структура поля течения остается практически неизменной. Однако влияние на распределение давления и плотности конвективного теплового потока весьма заметное, что проявляется при сравнении с экспериментальными данными.

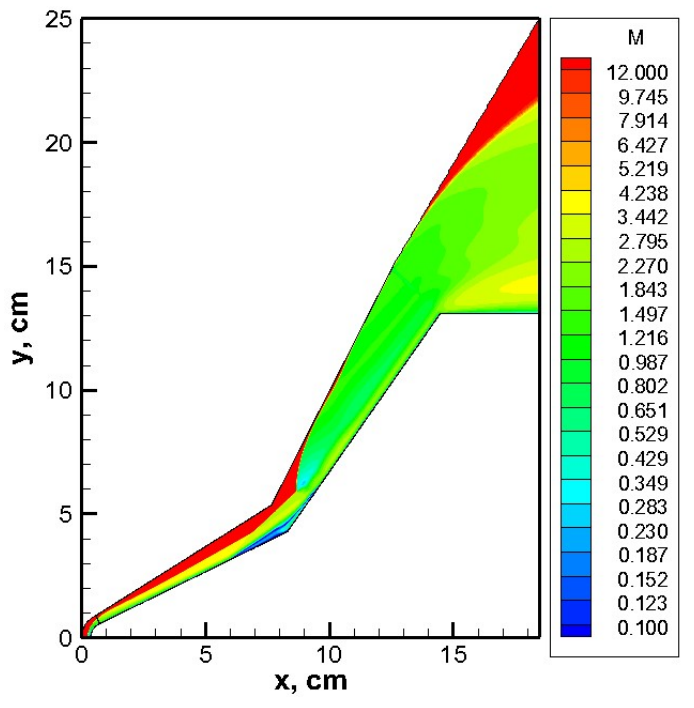

(a)

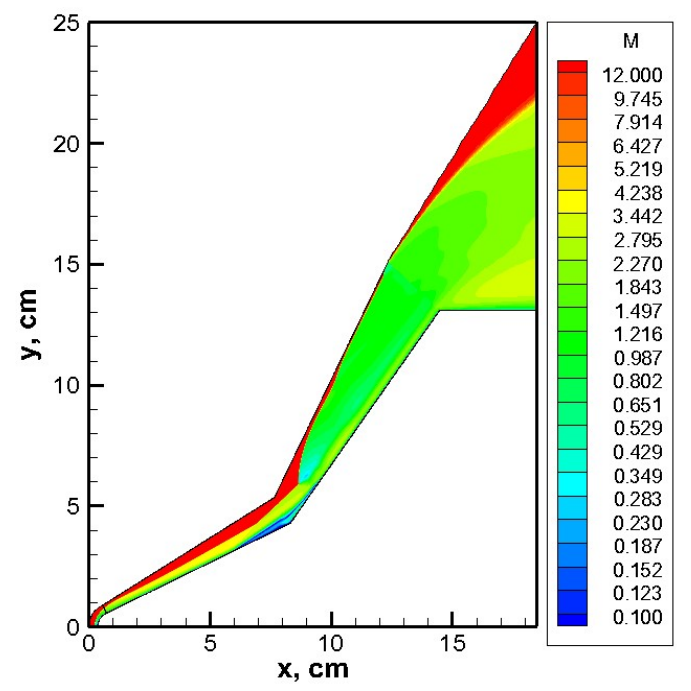

(b)

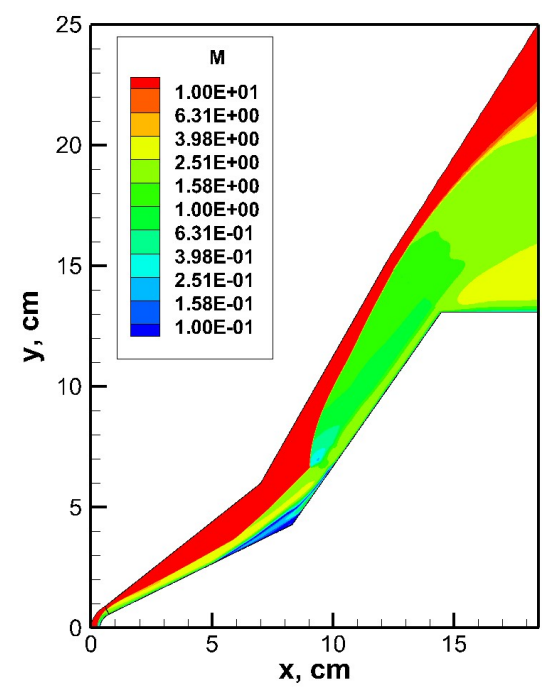

(б)

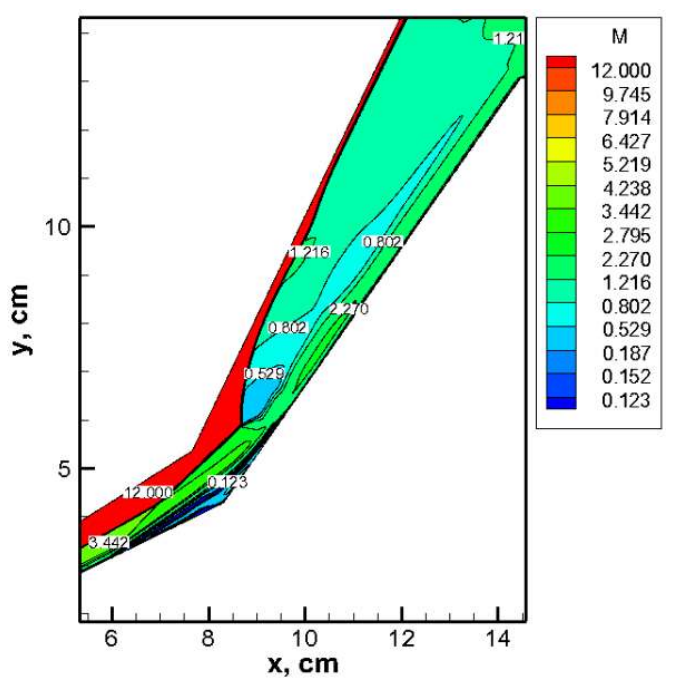

(2)

Рис. 10. Поле чисел Маха для тестовой задачи Run\#31 (расчетная сетка $703 \times 1505$, коэффициент сгущения расчетной сетки к поверхности $\beta=0.7(a), 0.6(\sigma)$ и $0.3($ (в)) 
Отметим особенность обтекания двойного затупленного конуса. Во-первых - это образование энтропийного слоя от затупления конуса. Это хорошо видно на распределении температуры (рис. 11) и модуля градиента плотности (рис. 14). Граница энтропийного слоя хорошо видна и на рис. 14 (кривая, отходящая от головной ударной волны). Граница энтропийного слоя простирается вплоть до скачка, генерируемого отрывным течением.

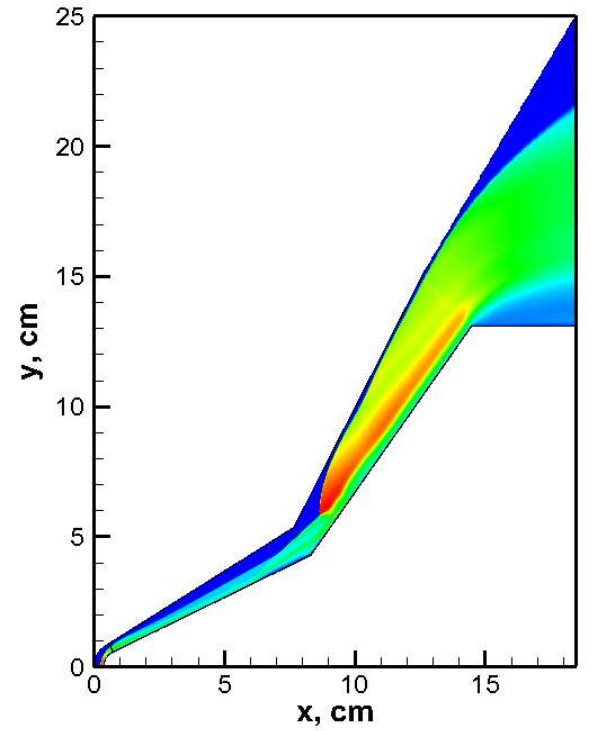

(a)

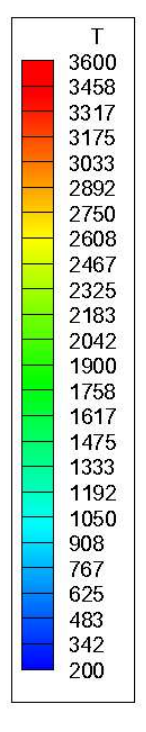

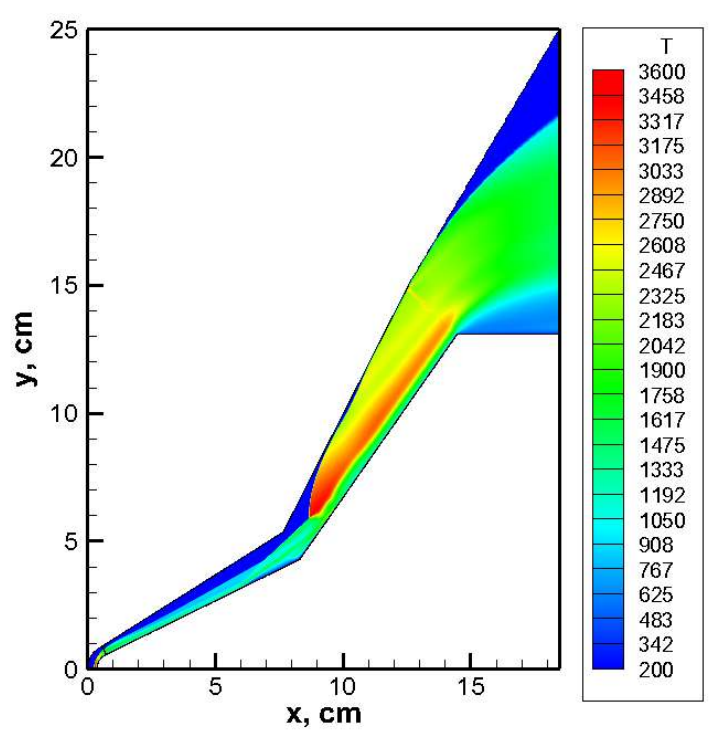

(б)

Рис. 11. Поле температуры (в K) для тестовой задачи Run\#31a (расчетная сетка $703 \times 1505$, коэффициент сгущения расчетной сетки к поверхности $\beta=0.8$ (a) и 0.7 (б))

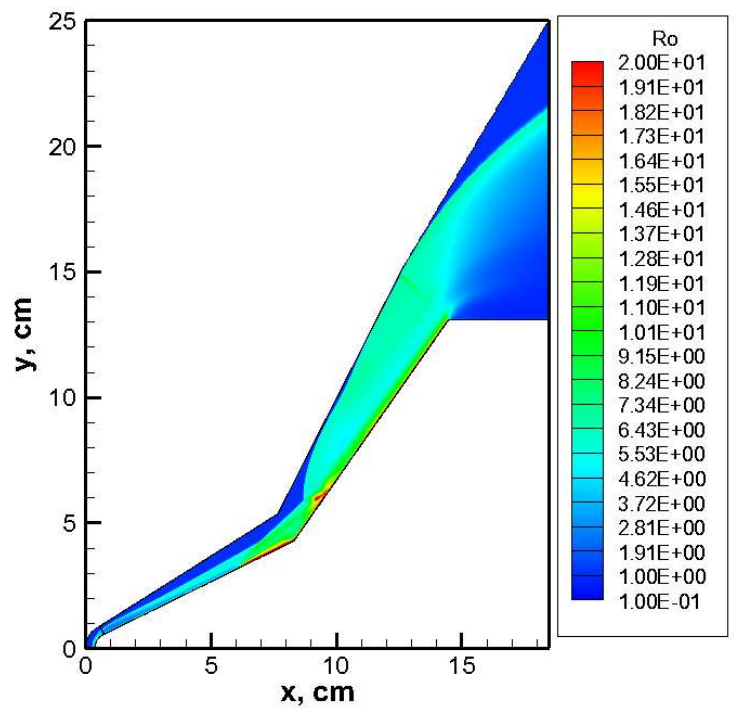

(a)

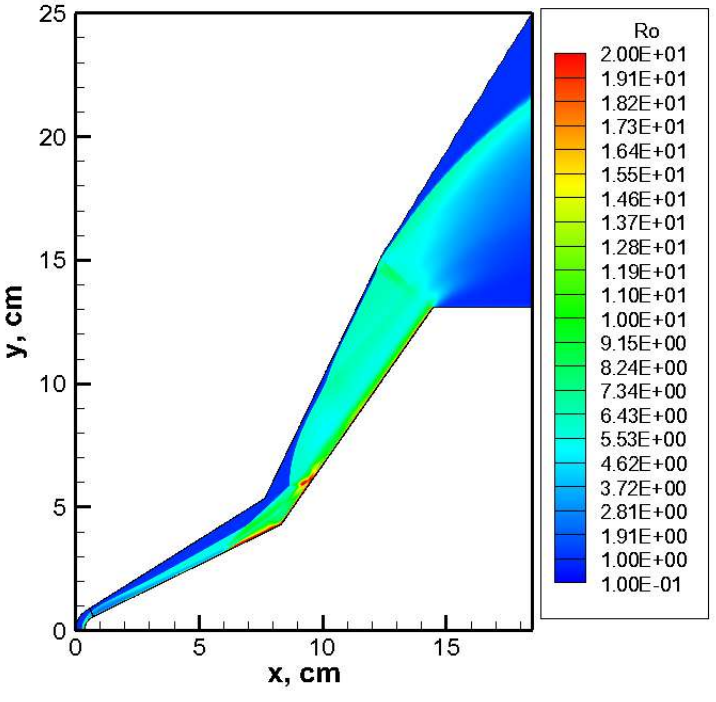

(б)

Рис. 12. Поле плотности $R_{0}=\rho / \rho_{\infty}$ для тестовой задачи Run\#31 (расчетная сетка $703 \times 1505$, коэффициент сгущения расчетной сетки к поверхности $\beta=0.7$ (a) и $0.3($ б))

Важной особенностью рассматриваемой тестовой задачи является существенно более сложная структура течения в области взаимодействия головной ударной волны с пограничным слоем $(x \sim 9 \div 10$ см). Отметим искривление головной ударной волны под действием скачка отрыва (рис. 14).

За областью взаимодействия отметим зону дозвукового движения за фронтом отраженной головной ударной волны. Ниже этой зоны находится подобласть сверхзвукового движения и, уже ближе к поверхности - область пограничного слоя (см. рис. 10). 
Примечательным элементом течения является высокотемпературная область дозвукового движения, где температура достигает порядка $3000 \mathrm{~K}$ (см. рис. 11). Вблизи поверхности двойного конуса отметим две зоны повышенной плотности: в области взаимодействия падающей ударной волны с пограничным слоем и в области отрывного течения (см. рис. 12).

На рис. 13 показаны распределения продольной скорости для трех вариантов степени сжатия расчетной сетки к поверхности. Местоположение внешней границы расчетной области тщательно подбиралось в численных экспериментах во избежание влияния на решение.

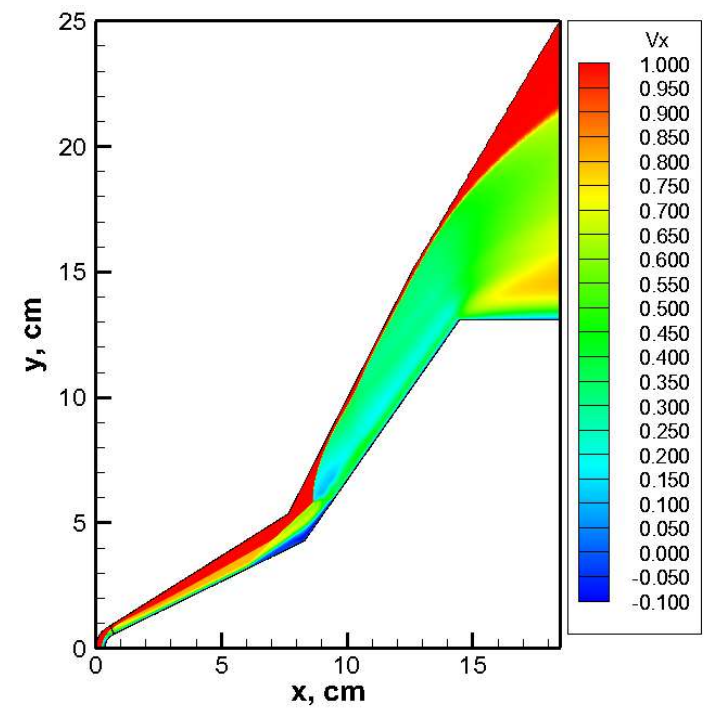

(a)

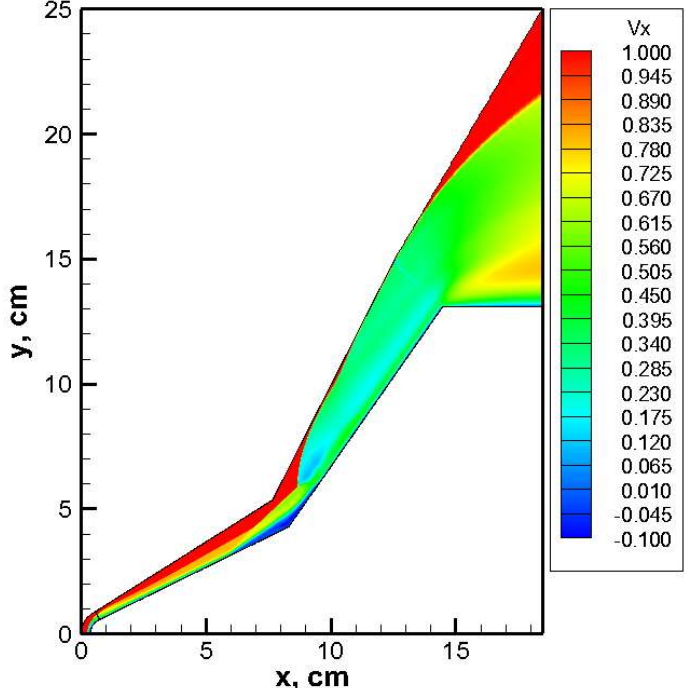

(б)

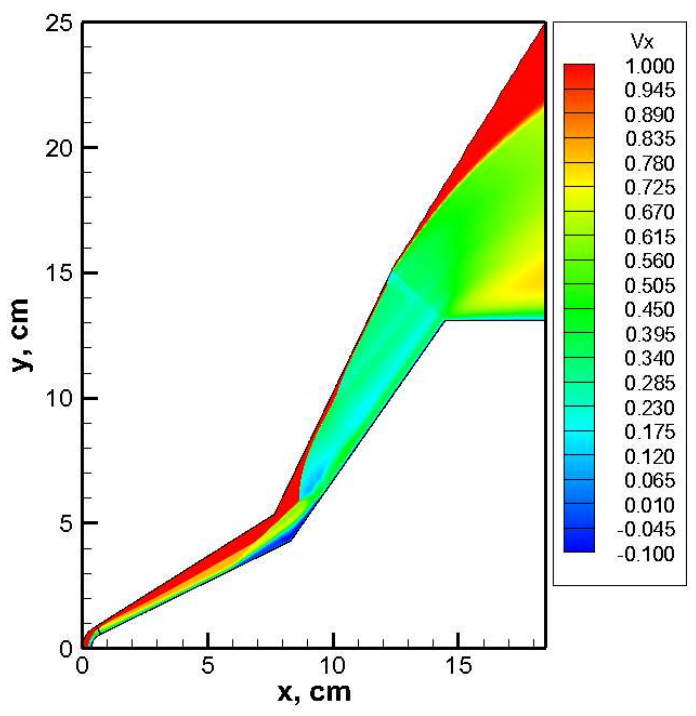

(b)

Рис. 13. Поле продольной скорости $V_{x}=u / V_{\infty}$ для тестовой задачи Run\#31 (расчетная сетка $703 \times 1505$, коэффициент сгущения расчетной сетки к поверхности $\beta=0.8(a)$, 0.7 (б) и $0.3($ (в))

Несмотря на малость видимых различий в профилях скорости, отметим, что такие различия видны на распределениях коэффициентов давления и теплообмена в сравнении с экспериментальными данными (рис. 15 и 16).

На рис. 17 показаны результаты расчета тестового варианта Run\#32, отличие которого от предыдущего варианта состоит в уменьшении скорости потока до $\mathrm{M}=10.6$. Давление в набегающем потоке примерно в два раза выше. 

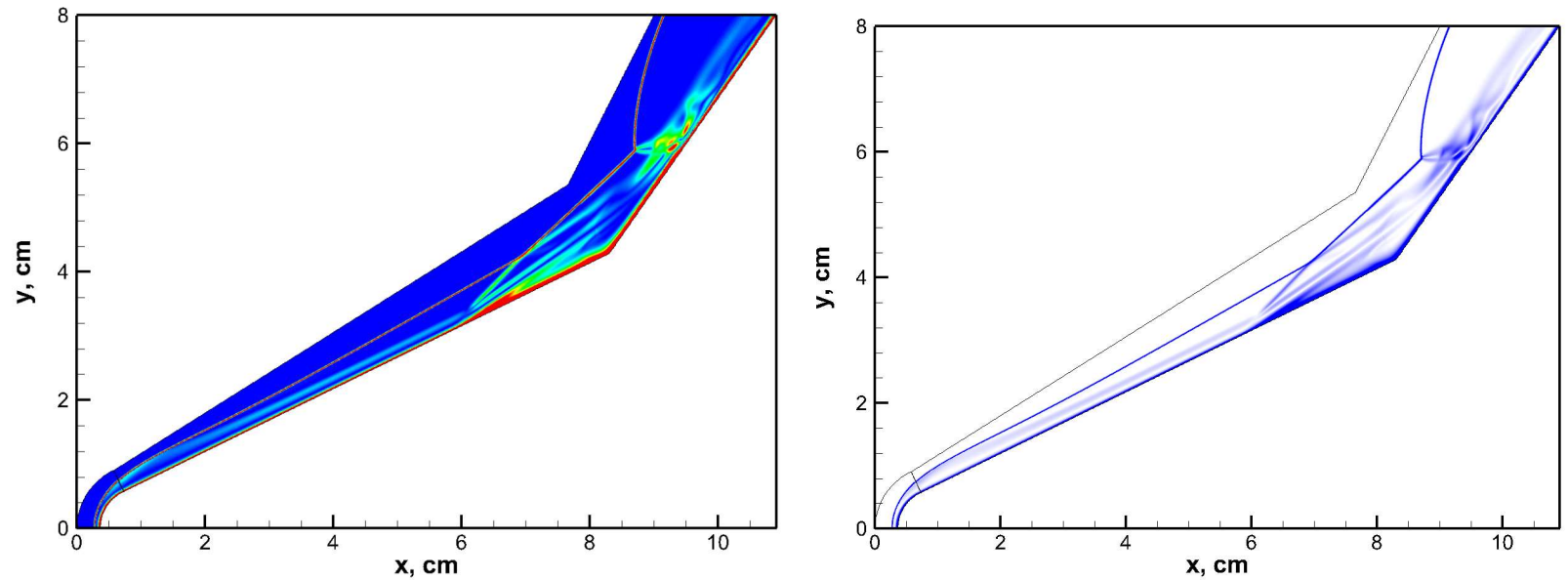

Рис. 14. Поле модуля градиента плотности для тестовой задачи Run\#31 (расчетная сетка $703 \times 1505$, коэффициент сгущения расчетной сетки к поверхности $\beta=0.7$ )

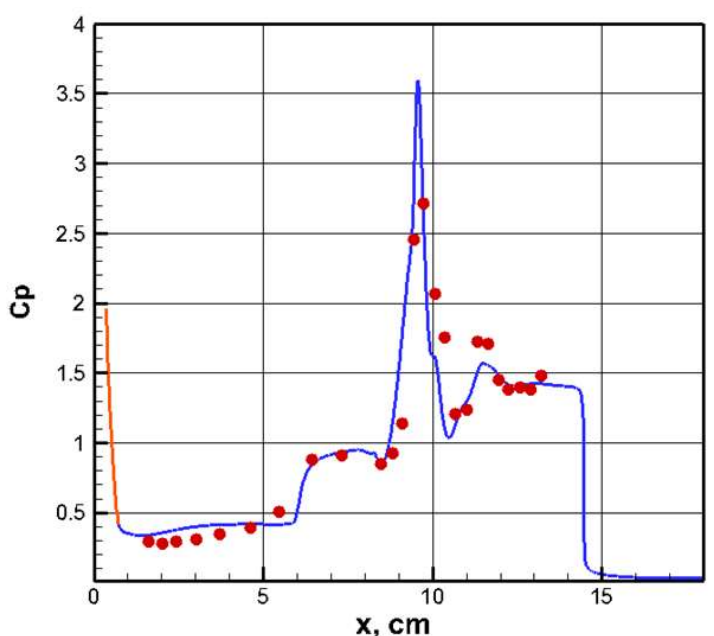

(a)

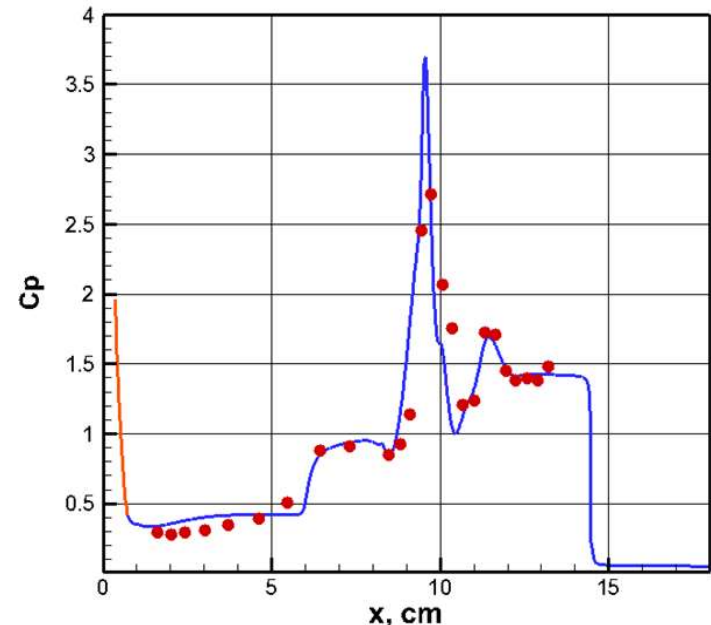

(б)

Рис. 15. Распределение коэффициента давления вдоль поверхности полого цилиндра с юбкой для тестовой задачи Run\#31 (расчетная сетка $703 \times 1505$, коэффициент сгущения расчетной сетки к поверхности $\beta=0.7$ (a) и $0.3($ б))

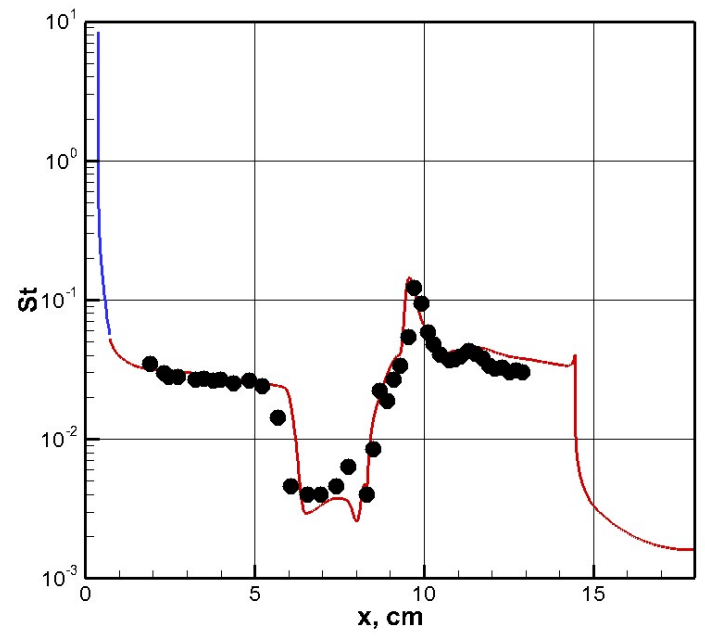

(a)

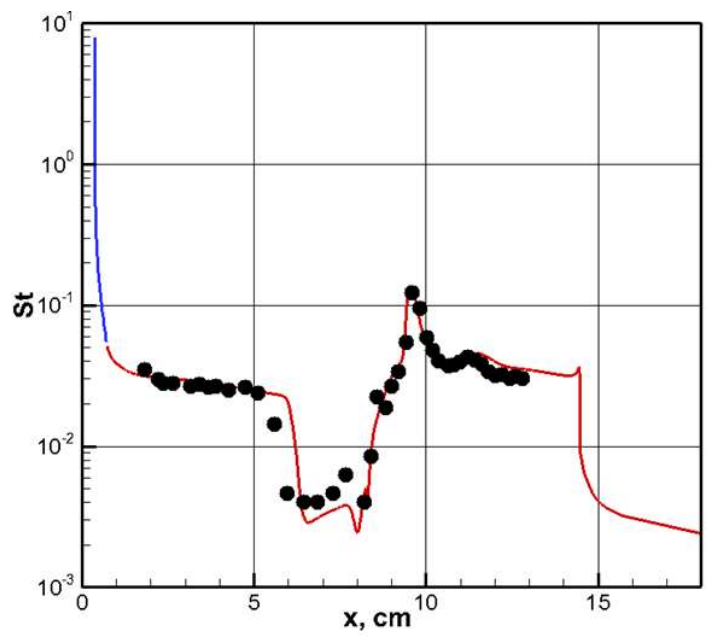

(б)

Рис. 16. Распределение коэффициента теплообмена St вдоль поверхности полого цилиндра с юбкой для тестовой задачи Run\#31 (расчетная сетка $703 \times 1505$, коэффициент сгущения расчетной сетки к поверхности $\beta=0.7$ (a) и $0.3($ б)) 
Особенности течения остаются подобными предыдущему тестовому случаю. Однако область отрывного течения здесь более протяженная (сравните рис. 16 и 19).

На рис. $17, a$ хорошо видна зона повышенного давления в области взаимодействия головной ударной волны с пограничным слоем (при $x \sim 10$ см) и зона повышенного давления над отрывным течением. Увеличенная область отрывного течения приводит к заметно более раннему, чем прежде, отклонению головной ударной волны от поверхности конуса (рис. 18). У обтекаемой поверхности наблюдается несколько областей сверх- и дозвукового движения. У лобового затупления формируется область дозвукового движения. Еще одна дозвуковая зона формируется в области отрывного течения. Дозвуковая зона наблюдается за отраженной ударной волной после взаимодействия с пограничным слоем. И, наконец, дозвуковое течение реализуется в пограничном слое вдоль всей обтекаемой поверхности.

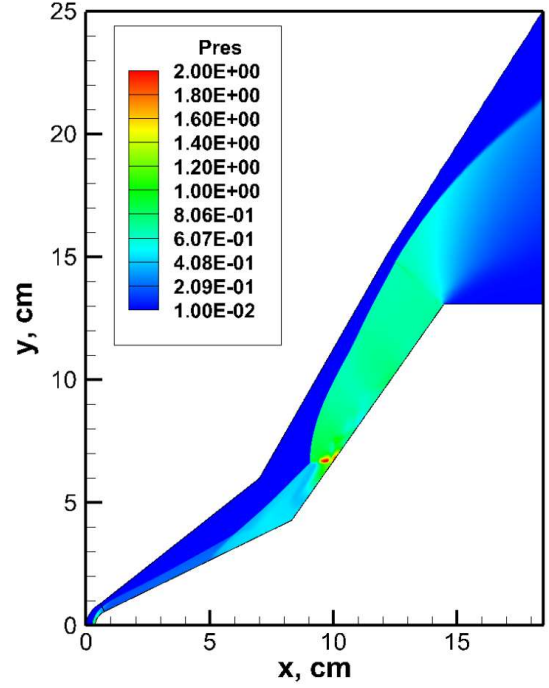

(a)

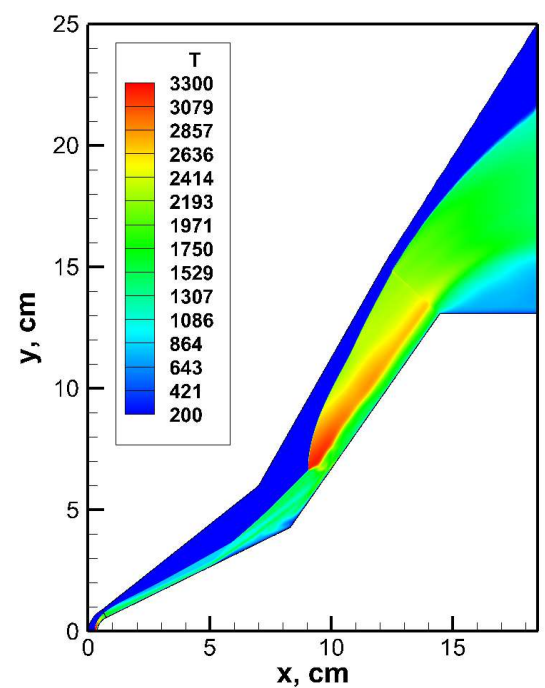

(б)

Рис. 17. Поле давления (Pres $\left.=p / \rho_{\infty} V_{\infty}^{2}\right)$ и температуры (в K) для тестовой задачи Run\#32 (расчетная сетка $703 \times 1505$, коэффициент сгущения расчетной сетки к поверхности $\beta=0.6)$

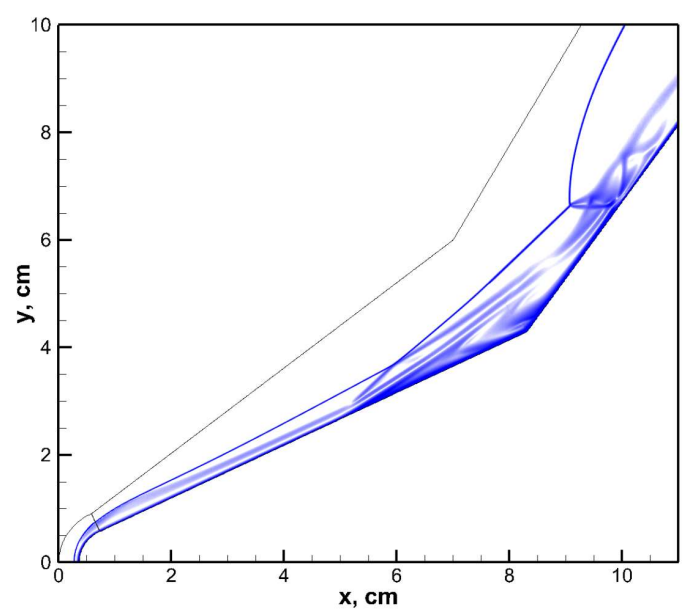

(a)

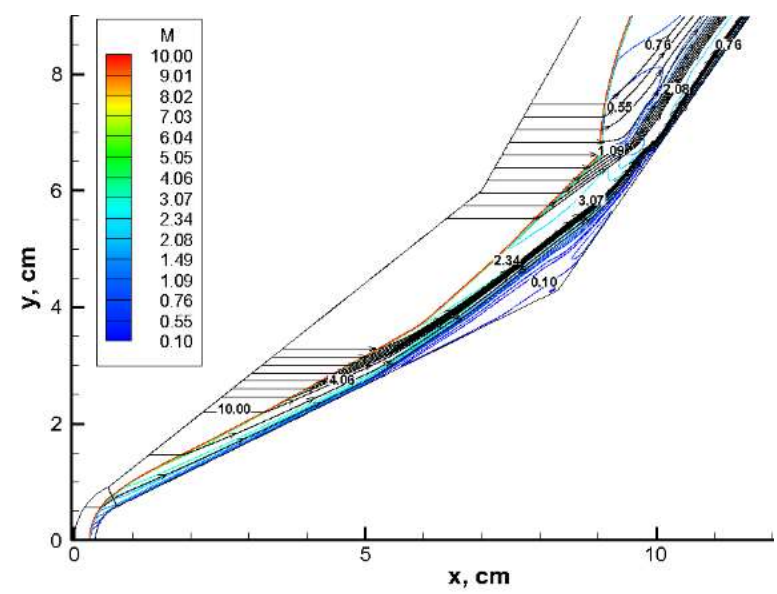

(б)

Рис. 18. Поле модуля градиента плотности (a) и чисел Маха (б) для тестовой задачи Run\#32 (расчетная сетка $703 \times 1505$, коэффициент сгущения расчетной сетки к поверхности $\beta=0.6$ )

Рассматриваемый тестовый вариант оказался весьма сложным для успешного описания экспериментальных данных. Как видно из рис. 19 в расчетах получается несколько меньшая протяженность зоны отрывного течения. 


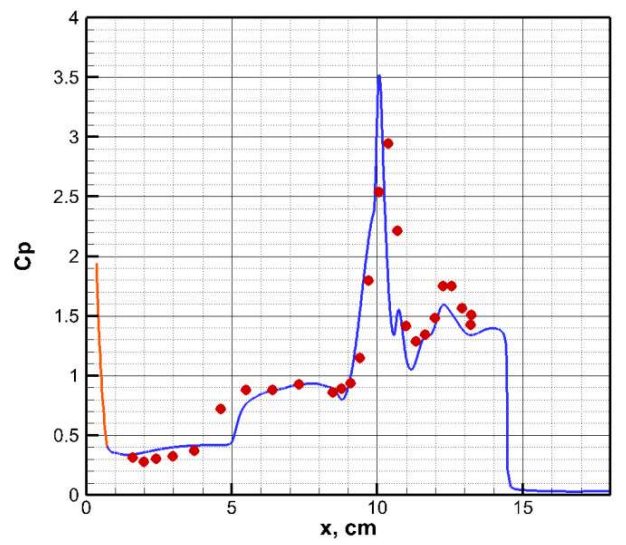

(a)

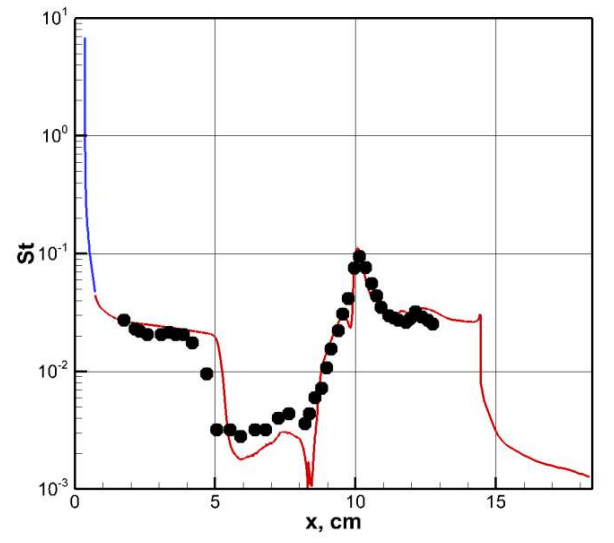

(б)

Рис. 19. Распределение коэффициента давления $C_{p}$ и теплообмена $\mathrm{St}$ вдоль поверхности двойного затупленного конуса для тестовой задачи Run\#32 (расчетная сетка $703 \times 1505$, коэффициент сгущения расчетной сетки к поверхности $\beta=0.6$ )

B тестовой задаче Run\#35 (рис. 20-22) изучалось обтекание двойного острого конуса. Структура поля течения в целом подобна рассмотренной выше, но отсутствие энтропийного слоя все скачки и переходные зоны более четкими.

На рис. 20 показаны распределения газодинамических функций для тестовой задачи Run\#35, а на рис. 21 представлено поле модуля градиента плотности и чисел Маха с линиями тока. Здесь также хорошо видны области сверх- и дозвукового движения. Рисунок 22 демонстрирует хорошее согласие расчетных и экспериментальных данных.

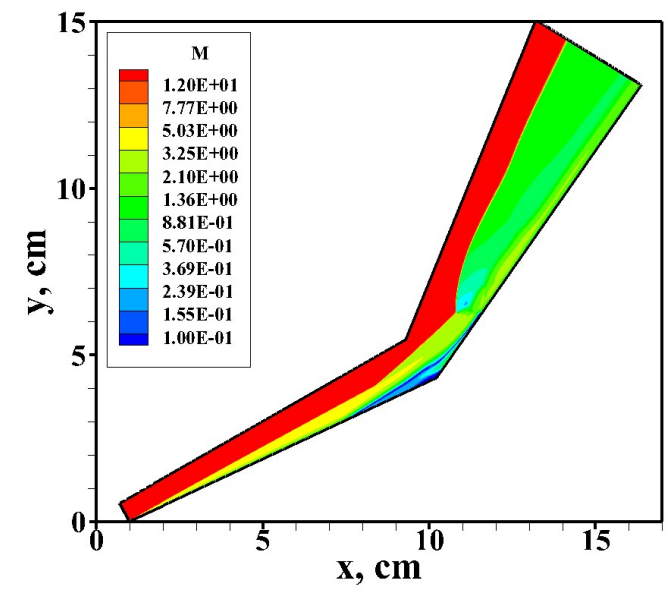

(a)

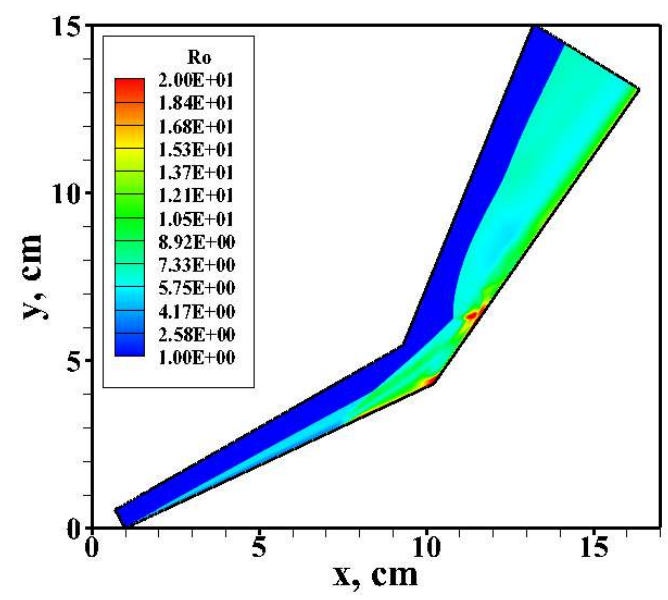

(b)

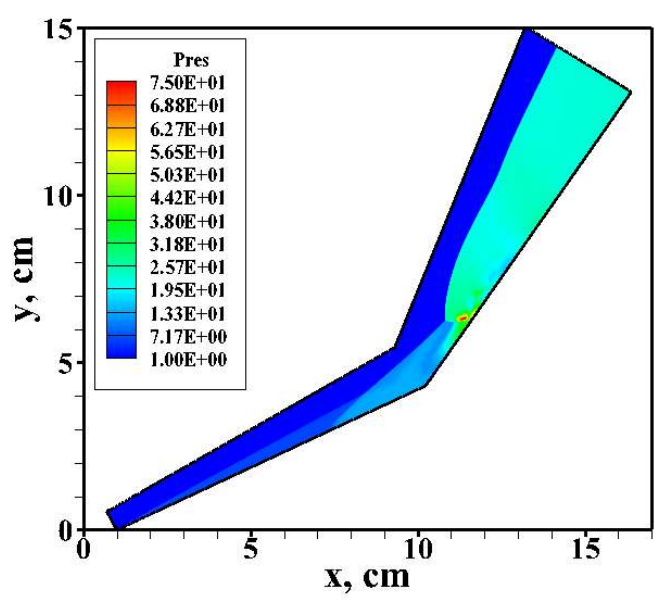

(б)

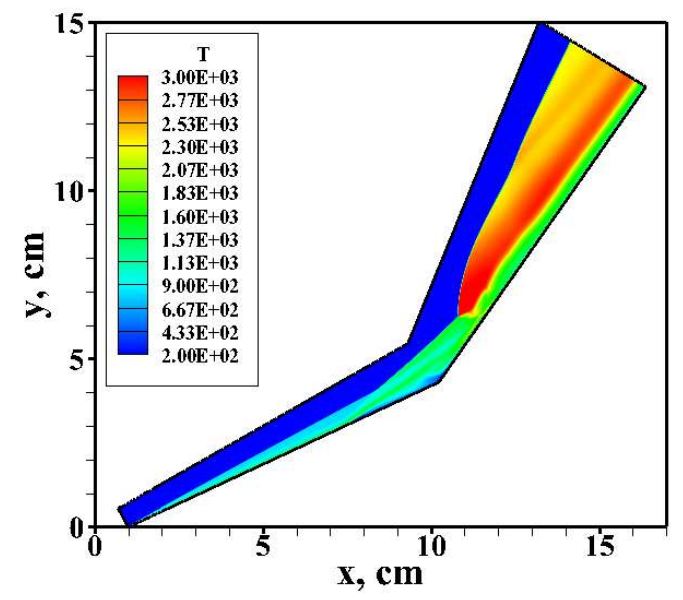

(2) 


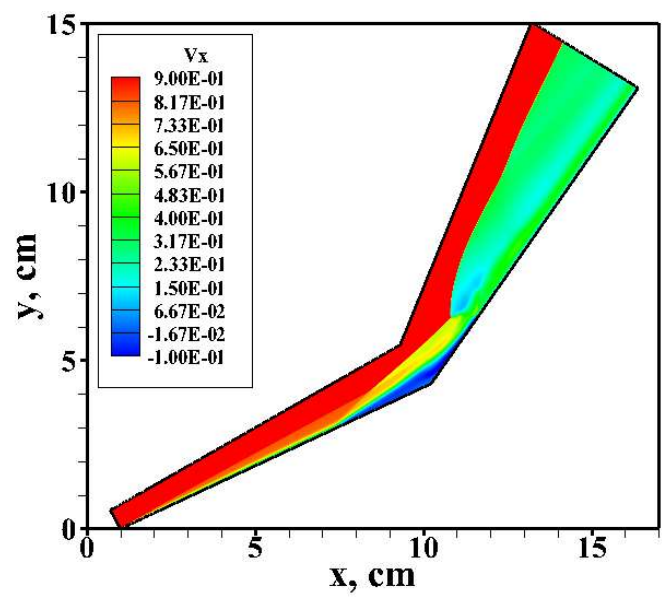

$(\partial)$

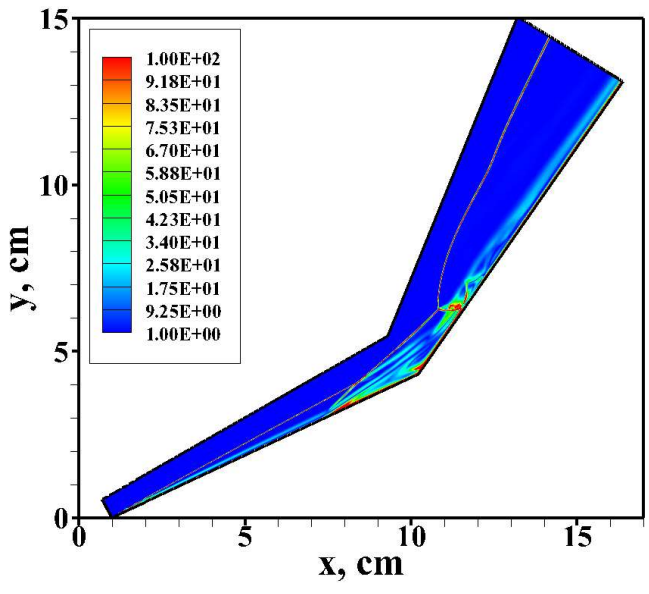

(e)

Рис. 20. Поле чисел Маха (a), давления ( $\left.\mathrm{Pres}=p / \rho_{\infty} V_{\infty}^{2}\right)$ (б), плотности (в), температуры (в К) (2), продольной скорости $V_{x}=u / V_{\infty}(\partial)$ и модуля градиента плотности (e) для тестовой задачи Run\#35 (расчетная сетка $385 \times 4001$, коэффициент сгущения расчетной сетки к поверхности $\beta=0.6$ )

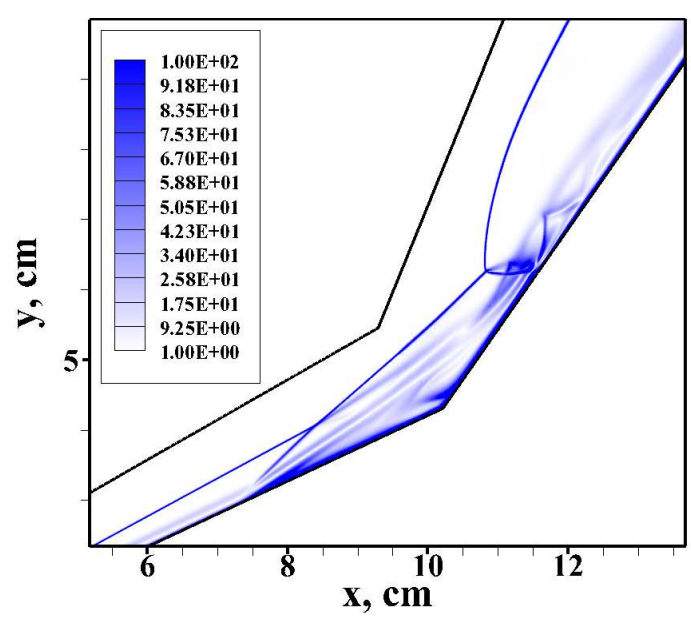

(a)

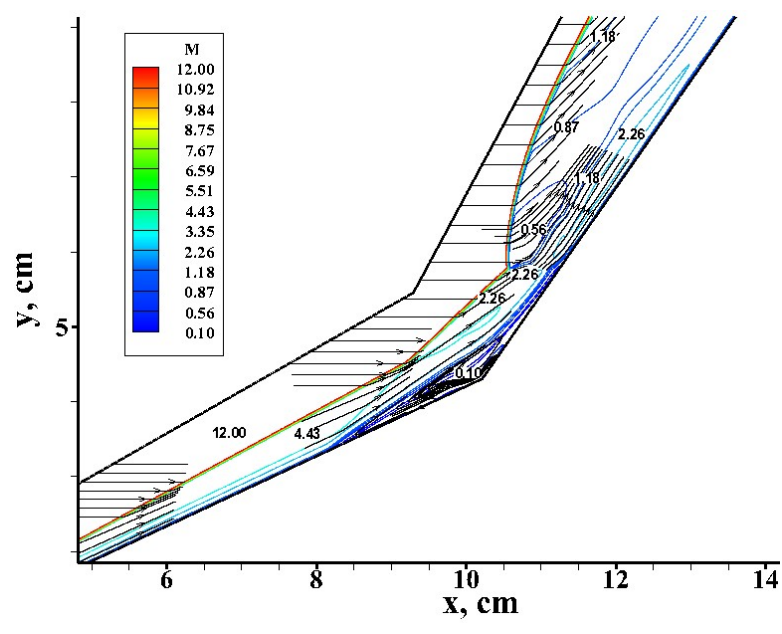

(б)

Рис. 21. Поле модуля градиента плотности (a) и чисел Маха (б) для тестовой задачи Run\#35 (расчетная сетка $385 \times 4001$, коэффициент сгущения расчетной сетки к поверхности $\beta=0.6$ )

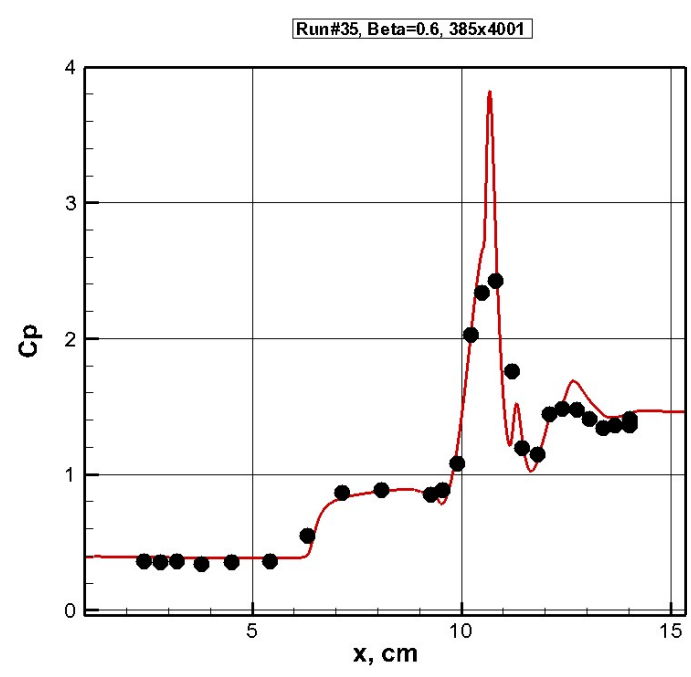

(a)

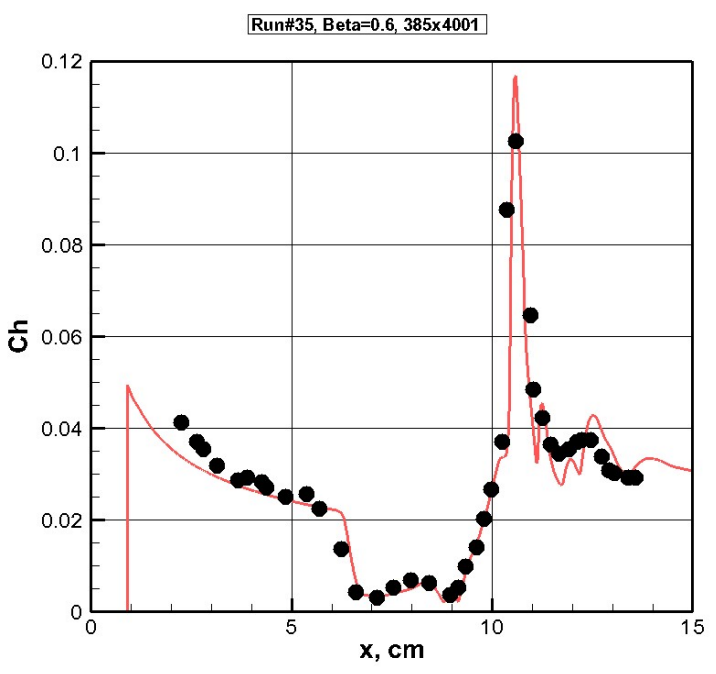

(б)

Рис. 22. Распределение коэффициента давления $C_{p}$ теплообмена St вдоль поверхности двойного острого конуса для тестовой задачи Run\#35 


\section{Заключение}

C использованием авторского компьютерного кода PERAT-2D выполнены валидационные расчеты ударно-волнового взаимодействия с ламинарным пограничным слоем при гиперзвуковом обтекании полого цилиндра с конической юбкой, острого и затупленного двойного конуса.

С использованием численного моделирования изучены экспериментальные данные, полученные в экспериментах Run\#11, 14, 31, 32 и 35 на гиперзвуковой аэродинамической трубе Calspan-UB Research Center (CUBRC) [1].

Показано хорошее согласие с экспериментальными данными по распределению давления и плотности конвективного теплового потока вдоль поверхности обтекаемых моделей.

В численных экспериментах изучено влияние расчетных параметров и геометрии расчетной области на степень согласия с экспериментальными данными. Показано, что для хорошего количественного совпадения расчетных и экспериментальных данных при использовании схем 2-го порядка аппроксимации, реализованных к коде PERAT-2D, требуются весьма подробные расчетные сетки, которые кроме хорошего описания экспериментальных данных, позволяют получить детальное разрешение газодинамической структуры течения при ударноволновом взаимодействии с пограничным слоем при гиперзвуковом обтекании.

Работа выполнена по теме государственного задания РАН (№ гос. регистрации ААААА20-120011690135-5) и частично при поддержке РФФИ (грант № 19-01-00515).

\section{Литература}

1. Holden M.S., Wadhams T.P., Harvey J.K., Candler G.V. Comparison between Measurements in Regions of Laminar Shock Wave Boundary Layer Interaction in Hypersonic Flows with Navier-Stokes and DSMC Solutions. RTO-TR-AVT-007-03.

2. Candler G.V., Nompelis I., Druguet M.-C., Holden M.S., Wadhams T.P., Boyd I.D. CFD Validation for Hypersonic Flight: Hypersonic Double-Cone Flow Simulation. AIAA 2002-0581. 14 p.

3. MacLean M., Holden M. Validation and Comparison of WIND and DPLR Results for Hypersonic, Laminar Problems. AIAA 2004-0529. 17 p.

4. Moss J.N., Olejniczak J. Shock-Wave/Boundary-Layer Interactions in Hypersonic Low Density Flows. AIAA 98-2668. 19 p.

5. Wright M.J., Sinha K., Olejniczak J., Candler G.V. Numerical and Experimental Investigation of Double-Cone Shock Interactions. AIAA Journal. 2000. Vol.38. No.12. pp. 2268-2276.

6. Harvey J.K., Holden M.S., Wadhams T.P. Code Validation Study of Laminar Shock/Boundary Layer and Shock/Shock Interactions in Hypersonic Flow. AIAA 2001-1031. 27 p.

7. Wang W.-L., Boyd I.D. Particle and Continuum Computations of Hypersonic Flow over Sharp and Blunted Cones. AIAA 2001-2900. 12 p.

8. Kato H., Tannehill J.C. Computation of Hypersonic Laminar Separated Flows using an Iterated PNS Algorithm. AIAA 2001-1028. $16 \mathrm{p}$.

9. Candler G.V., Druguet M.-C. Navier-Stokes Predictions of Hypersonic Double-Cone and CylinderFlare Flow Fields. AIAA 2001-1024. 10 p.

10. Gnoffo P.A. CFD Validation Studies for Hypersonic Flow Prediction. AIAA 2001-1025. 13 p.

11. Roy C.J., Gallis M.A., Bartel T.J., Payne J.L. Navier-Stokes and DSMC Simulations for Hypersonic Laminar Shock-Shock Interaction Flows. AIAA 2002-0737. 16 p.

12. Harvey J.K., Holden M.S., Candler G.V. Validation of DSNC/Navier-Stokes Computations for Laminar Shock Wave/Boundary Layer Interactions in Hypersonic Flows. AIP Proceedings 2003. 663. pp. $417-424$.

13. Moss J.N., Bird G.A. DSMC Simulations of Hypersonic Flows with Shock Interactions and Validation with Experiments. 2004. AIAA 2004-2585. 13 p. 
14. MacLean M., Candler G., Holden M. Numerical Evaluation of Flow Conditions in the LENS Reflected Shock-Tunnel Facilities. 2005. AIAA 2005-0903. 14 p.

15. Knight D. RTO WG 10: Test Cases for CFD Validation of Hypersonic Flight. RTO-TR-AVT-007-V3. $24 \mathrm{p}$.

16. MacLean M., Holden M., Wadhams T., Parker R. A computational Analysis of Thermochemical Studies in the LENA Facilities. 2007. AIAA 2007-0121. 16 p.

17. MacLean M., Mundy E., Wadhams T., Holden M., Johnson H., Candler G. Comparisons of Transition Prediction using PSE-Chem to Measurements for a Shock Tunnel Environment. 2007. AIAA 20074490. $18 \mathrm{p}$.

18. Shen Y., Zha G., Huerta M.A., Gables C. Simulation of Hypersonic Shock Wave/Boundary Layer Interaction Using High Order WENO Scheme. 2010. AIAA 2010-1047. 14 p.

19. Holden M., MacLean M., Wadhams T., Mundy E. Experimental Studies of Shock Wave/Turbulent Boundary Layer Interactions in High Reynolds Number Supersonic and Hypersonic Flows to Evaluate the Performance of CFD Codes. 2010. AIAA 2010-4468. 23 p.

20. Jing 1., Hong X., Di W. Simulation and Experimental Validation of Hypersonic Shock Wave Interaction. Research Journal of Applied Sciences, Engineering and Technology. 2013. 6(22) pp.4221-4224.

21. Hao J., Wang J., Lee C. Numerical Simulation of High-Enthalpy Double-Cone Flows. AIAA J. 2017. Vol.55. No.5. pp.2471-2475.

22. Walker S., Schmisseur J.D. CFD Validation of Shock-Shock Interaction Flow fields. 2002. AIAA 20020436. $22 \mathrm{p}$

23. Candler G.V., Nompelis I. CFD Validation for Hypersonic Flight: Real Gas Flows. AIAA 2002-0434. 2002. 9 p.

24. Knight D., Hong Y., Panaras A., Zheltovodov A. RTO WG 10: CFD Validation for Shock Wave Turbulent Boundary Layer Interactions. AIAA 2002-0437. 2002. 30 p.

25. Holden M., MacLean M., Wadhams T., Dufrene A. Measurements of Real Gas Effects on Regions of Laminar Shock Wave/Boundary Layer Interaction in Hypervelocity Flows for "Blind" Code Validation Studies. AIAA 2013-2837. 2013. 13 p.

26. Боровой В.Я., Егоров И.В., Мошаров В.Е., Скуратов А.С., Радченко В.Н. Экстремальный нагрев тел в гиперзвуковом потоке. М.: Наука. 2018. 390 с.

27. Боровой В.Я., Скуратов А.С., Струминская И.В. О существовании «пороговой» величины притупления пластины при интерференции косого скачка уплотнения с пограничным и энтропийным слоями//Изв. РАН МЖГ. 2008. № 3. С.41-52.

28. Боровой В.Я., Егоров И.В., Скуратов А.С., Струминская И.В. Ламинарный теплообмен острых и притупленных пластин в гиперзвуковом потоке воздуха//Изв. РАН МЖГ. 2005. №1. С.168-180.

29. Shang J.S., Surzhikov S.T. Magnetoaerodynamic Actuator for Hypersonic Flow Control// AIAA J. 2005. Vol. 43, No. 8. Pp. 1633-1643.

30. Shang J.S., Surzhikov S.T., Kimmel R., Gaitonde D., Menart J., Hayes J. Mechanisms of plasma actuators for hypersonic flow control// Progress in Aerospace Sciences. 2005. Vol. 41. Pp. 642-668.

31. Surzhikov S.T. Theoretical and Computational Physics of Gas Discharge Phenomena. Walter de Gruyter $\mathrm{GmbH}, 2020.549$ p.

32. Menart J., Shang J. S., Kimmel R., and Hayes J. Effect of Magnetic Fields on Plasma Generated in a Mach 5 Wind Tunnel," AIAA Paper 2003-4165, June 2003.

33. Shang J., Kimmel R., Menart J., Surzhikov S. Hypersonic Flow Control Using Surface Plasma Actuator//Journal of Propulsion and Power. Vol. 24, No. 5, pp. 923-934.

34. Surzhikov S.T. Surface electromagnetic actuator in rarefied hypersonic flow// IOP Conf. Series: Journal of Physics: Conf. Series 815 (2017) 012005 doi:10.1088/1742-6596/815/1/012005

35. Крючкова А.С. Моделирование сверхзвукового обтекания баллистических моделей в программном коде UST3D// Физико-химическая кинетика в газовой динамике. 2018. T.19 (4) http://chemphys.edu.ru/issues/2018-19-4/articles/783/ 
36. Edwards, J.R., Liou, M.-S. Low-Diffusion Flux-Splitting Methods for Flow at all Speeds // AIAA Journal. 1998. Vol. 36. № 9. Pp. 1610-1617.

\section{References}

1. Holden, M. S., Wadhams, T.P., Harvey, J. K., Candler, G. V., "Comparison between Measurements in Regions of Laminar Shock Wave Boundary Layer Interaction in Hypersonic Flows with Navier - Stokes and DSMC Solutions," RTO-TR-AVT-007-03.

2. Candler, G. V., Nompelis, I., Druguet, M.-C., Holden, M. S., Wadhams, T. P., Boyd, I. D., "CFD Validation for Hypersonic Flight: Hypersonic Double-Cone Flow Simulation," 40th AIAA Aerospace Sciences Meeting \& Exhibit, AIAA Paper 2002-0581, Jan. 2002.

3. MacLean, M., Holden, M., "Validation and Comparison of WIND and DPLR Results for Hypersonic, Laminar Problems," 42th AIAA Aerospace Sciences Meeting \& Exhibit, AIAA Paper 2004-0529, Jan. 2004.

4. Moss, J. N., Olejniczak, J., "Shock-Wave/Boundary-Layer Interactions in Hypersonic Low Density Flows," 7th AIAA/ASME Joint Thermophysics and Heat Transfer Conference, AIAA Paper 98-2668, June 1998.

5. Wright, M. J., Sinha, K., Olejniczak, J., Candler G. V., "Numerical and Experimental Investigation of Double-Cone Shock Interactions," AIAA Journal, Vol. 38, No. 12, Dec. 2000, pp. 2268-2276.

doi:10.2514/2.918

6. Harvey, J. K., Holden, M. S., Wadhams, T. P., "Code Validation Study of Laminar Shock/Boundary Layer and Shock/Shock Interactions in Hypersonic Flow," 39th Aerospace Sciences Meeting and Exhibit, AIAA Paper 2001-1031, Jan. 2001.

7. Wang, W.-L., Boyd, I. D., "Particle and Continuum Computations of Hypersonic Flow over Sharp and Blunted Cones," 35th AIAA Thermophysics Conference, AIAA Paper 2001-2900, June 2001.

8. Kato, H., Tannehill, J. C., "Computation of Hypersonic Laminar Separated Flows using an Iterated PNS Algorithm," 39th Aerospace Sciences Meeting \& Exhibit, AIAA Paper 2001-1028, Jan. 2001.

9. Candler, G. V., Druguet, M.-C., "Navier-Stokes Predictions of Hypersonic Double-Cone and CylinderFlare Flow Fields," 39th AIAA Aerospace Sciences Meeting \& Exhibit, AIAA Paper 2001-1024, 2001.

10. Gnoffo, P. A., "CFD Validation Studies for Hypersonic Flow Prediction," 39th AIAA Aerospace Sciences Meeting \& Exhibit, AIAA Paper 2001-1025, Jan. 2001.

11. Roy C. J., Gallis M. A., Bartel T. J., Payne J. L., "Navier-Stokes and DSMC Simulations for Hypersonic Laminar Shock-Shock Interaction Flows," AIAA 2002-0737, 2002.

12. Harvey, J. K., Holden, M. S., Candler, G. V., "Validation of DSNC/Navier-Stokes Computations for Laminar Shock Wave/Boundary Layer Interactions in Hypersonic Flows,” AIP Proceedings, 663, 2003, pp.417-424.

13. Moss, J. N., Bird, G. A., "DSMC Simulations of Hypersonic Flows with Shock Interactions and Validation with Experiments," 37th AIAA Thermophysics Conference, AIAA Paper 2004-2585, June 2004.

14. MacLean, M., Candler, G., Holden, M., "Numerical Evaluation of Flow Conditions in the LENS Reflected Shock-Tunnel Facilities," 43rd AIAA Aerospace Sciences Meeting, AIAA Paper 2005-0903, Jan. 2005.

15. Knight, D., "RTO WG 10: Test Cases for CFD Validation of Hypersonic Flight," RTO-TR-AVT-007$\mathrm{V} 3,24 \mathrm{p}$.

16. MacLean, M., Holden, M., Wadhams, T., Parker, R., "A computational Analysis of Thermochemical Studies in the LENA Facilities," 45TH AIAA Aerospace Sciences Meeting \& Exhibit, AIAA Paper 20070121, Jan. 2007.

17. MacLean, M., Mundy, E., Wadhams, T., Holden, M., Johnson, H., Candler, G., "Comparisons of Transition Prediction using PSE-Chem to Measurements for a Shock Tunnel Environment," 37th AIAA Fluid Dynamics Conference and Exhibit, AIAA Paper 2007-4490, June 2007. 
18. Shen, Y., Zha, G., Huerta, M. A., Gables, C., "Simulation of Hypersonic Shock Wave/Boundary Layer Interaction Using High Order WENO Scheme," 48th AIAA Aerospace Sciences Meeting Including the New Horizons Forum and Aerospace Exposition, AIAA Paper 2010-1047. Jan. 2010.

19. Holden, M., MacLean, M., Wadhams, T., Mundy, E., "Experimental Studies of Shock Wave/Turbulent Boundary Layer Interactions in High Reynolds Number Supersonic and Hypersonic Flows to Evaluate the Performance of CFD Codes," 40th Fluid Dynamics Conference and Exhibit, AIAA Paper 20104468, June 2010.

20. Jing, 1., Hong, X., Di, W., "Simulation and Experimental Validation of Hypersonic Shock Wave Interaction. Research Journal of Applied Sciences," Engineering and Technology, 6(22), 2013, pp. 4221-4224.

21. Hao, J., Wang, J., Lee, C., "Numerical Simulation of High-Enthalpy Double-Cone Flows," AIAA Journal, Vol.55, No.5, Aug. 2017, pp. 2471-2475. doi:10.2514/1.J056643

22. Walker, S., Schmisseur, J. D., "CFD Validation of Shock-Shock Interaction Flow fields," 40th AIAA Aerospace Sciences Meeting \& Exhibit, AIAA Paper 2002-0436, Jan. 2002.

23. Candler, G.V., Nompelis, I., "CFD Validation for Hypersonic Flight: Real Gas Flows," 40th AIAA Aerospace Sciences Meeting \& Exhibit, AIAA Paper 2002-0434, Jan. 2002.

24. Knight, D., Hong, Y., Panaras, A., Zheltovodov, A., "RTO WG 10: CFD Validation for Shock Wave Turbulent Boundary Layer Interactions," 40th AIAA Aerospace Sciences Meeting \& Exhibit, AIAA Paper 2002-0437, Jan. 2002.

25. Holden, M., MacLean, M., Wadhams, T., Dufrene, A., "Measurements of Real Gas Effects on Regions of Laminar Shock Wave/Boundary Layer Interaction in Hypervelocity Flows for "Blind" Code Validation Studies," 21st AIAA Computational Fluid Dynamics Conference, AIAA Paper 2013-2837, June 2013.

26. Borovoj, V. Ja., Egorov, I. V., Mosharov, V. E., Skuratov, A. S., Radchenko, V. N., Jekstremal'nyj nagrev tel v giperzvukovom potoke (Extreme heating of bodies in hypersonic flow), M.: Nauka, 2018, $390 \mathrm{~s}$.

27. Borovoj, V. Ja., Skuratov, A. S., Struminskaja, I. V., "O sushhestvovanii «porogovoj» velichiny prituplenija plastiny pri interferencii kosogo skachka uplotnenija s pogranichnym i jentropijnym slojami," Izv. RAN MZhG, No 3, 2008, s. 41-52.

28. Borovoj V. Ja., Egorov I. V., Skuratov A. S., Struminskaja I. V., "Laminarnyj teploobmen ostryh i prituplennyh plastin v giperzvukovom potoke vozduha," Izv. RAN MZhG, No. 1, 2005, s.168-180.

29. Shang, J. S., Surzhikov, S. T., "Magnetoaerodynamic Actuator for Hypersonic Flow Control," AIAA Journal, Vol. 43, No. 8, 2005, pp. 1633-1643.

30. Shang, J. S., Surzhikov, S. T., Kimmel, R., Gaitonde, D., Menart, J., Hayes, J., "Mechanisms of plasma actuators for hypersonic flow control," Progress in Aerospace Sciences, Vol. 41, 2005, pp. 642-668.

31. Surzhikov, S. T., Theoretical and Computational Physics of Gas Discharge Phenomena, Walter de Gruyter GmbH, 2020, 549 p.

32. Menart, J., Shang, J. S., Kimmel, R., and Hayes, J., "Effect of Magnetic Fields on Plasma Generated in a Mach 5 Wind Tunnel," 34th AIAA Plasmadynamics and Lasers Conference, AIAA Paper 2003-4165, June 2003.

33. Shang, J., Kimmel, R., Menart, J., Surzhikov, S., "Hypersonic Flow Control Using Surface Plasma Actuator," Journal of Propulsion and Power, Vol. 24, No. 5, pp. 923-934.

34. Surzhikov, S. T., "Surface electromagnetic actuator in rarefied hypersonic flow," IOP Conf. Series: Journal of Physics: Conf. Series 815 (2017) 012005 doi:10.1088/1742-6596/815/1/012005

35. Krjuchkova A. S., "Modelirovanie sverhzvukovogo obtekanija ballisticheskih modelej v programmnom kode UST3D," Fiziko-himicheskaja kinetika v gazovoj dinamike (Physical-Chemical Kinetics in Gas Dynamics), Vol. 19, No. 4, 2018. http://chemphys.edu.ru/issues/2018-19-4/articles/783/

36. Edwards, J. R., Liou, M.-S., "Low-Diffusion Flux-Splitting Methods for Flow at all Speeds," AIAA Journal, Vol. 36, No. 9, Sept. 1998, pp. 1610-1617. doi:10.2514/2.587 